\title{
Growth of perturbations in an expanding universe with Bose-Einstein condensate dark matter
}

\begin{abstract}
P. H. Chavanis
Laboratoire de Physique Théorique (IRSAMC), CNRS and UPS, Université de Toulouse, France

e-mail: chavanis@irsamc.ups-tlse.fr

Received 16 March 2011 / Accepted 27 October 2011

\section{ABSTRACT}

We study the growth of perturbations in an expanding Newtonian universe with Bose-Einstein condensate (BEC) dark matter. We first ignore special relativistic effects and derive a differential equation that governs the evolution of the density contrast in the linear regime. This equation, which takes quantum pressure and self-interaction into account, can be solved analytically in several cases. We argue that an attractive self-interaction can enhance the Jeans instability and fasten the formation of structures. Then, we take pressure effects (coming from special relativity) into account in the evolution of the cosmic fluid and add the contribution of radiation, baryons, and dark energy (cosmological constant). For BEC dark matter with repulsive self-interaction (positive pressure) the scale factor increases more rapidly than in the standard $\Lambda \mathrm{CDM}$ model where dark matter is pressureless, while it increases less rapidly for BEC dark matter with attractive self-interaction (negative pressure). We study the linear development of the perturbations in these two cases and show that the perturbations grow faster in BEC dark matter than in pressureless dark matter. Finally, we consider a "dark fluid" with a generalized equation of state $p=\left(\alpha \rho+k \rho^{2}\right) c^{2}$ having a component $p=k \rho^{2} c^{2}$ similar to BEC dark matter and a component $p=\alpha \rho c^{2}$ mimicking the effect of the cosmological constant (dark energy). We find optimal parameters that give good agreement with the standard $\Lambda \mathrm{CDM}$ model that assumes a finite cosmological constant.
\end{abstract}

Key words. hydrodynamics - instabilities - dark matter - cosmology: theory - dark energy - methods: analytical

\section{Introduction}

Several astrophysical observations of distant type Ia supernovae have revealed that the content of the universe is made of about $70 \%$ dark energy, $25 \%$ dark matter, and $5 \%$ baryonic (visible) matter (Riess et al. 1998; Perlmutter et al. 1999; de Bernardis et al. 2000; Hanany et al. 2000). Thus, the overwhelming preponderance of matter and energy in the universe is believed to be dark, i.e. unobservable by telescopes. The dark energy is responsible for the accelerated expansion of the universe. Its origin is mysterious and presumably related to the cosmological constant. Dark energy is usually interpreted as a vacuum energy, and it behaves like a fluid with negative pressure. Dark matter is also mysterious. The suggestion that dark matter may constitute a large part of the universe was raised by Zwicky in 1933. Using the virial theorem to infer the average mass of galaxies within the Coma cluster, he obtained a much higher value than the mass of luminous material. He realized therefore that some mass was "missing" to account for observations. This missing mass problem was confirmed later by accurate measurements of rotation curves of disk galaxies (Rubin et al. 1980; Persic et al. 1996). The rotation curves of neutral hydrogen clouds in spiral galaxies measured from the Doppler effect are found to be roughly flat (instead of Keplerian) with a typical rotational velocity $v_{\infty} \sim 200 \mathrm{~km} \mathrm{~s}^{-1}$ up to the maximum observed radius of about $50 \mathrm{kpc}$. The corresponding mass profile is much more extended than the distribution of starlight, which typically converges within $\sim 10 \mathrm{kpc}$. This implies that galaxies are surrounded by an extended halo of dark matter, whose mass $M(r) \sim r v_{\infty}^{2} / G$ increases linearly with radius. This can be conveniently modeled by a classical isothermal gas whose density decreases as $r^{-2}$ (Chandrasekhar 1939). Although some authors like Milgrom (1983) propose a modification of Newton's law (MOND theory) to explain the rotation curves of spiral galaxies without invoking dark matter, the dark matter hypothesis is favored by most astrophysicists.

The nature of dark matter (DM) is one of the most important puzzles in modern physics and cosmology. A wide "zoology" of exotic particles that could form dark matter has been proposed. In particular, many grand unified theories in particle physics predict the existence of various exotic bosons (e.g. axions, scalar neutrinos, neutralinos) that should be present in considerable abundance in the universe and comprise (part of) the cosmological missing mass (Primack et al. 1998; Overduin \& Wesson 2004). Even if the bosonic particles have never been detected in accelerator experiments, they are considered as leading candidates of dark matter that might play a significant role in the evolution and structure of the universe.

If dark matter is made of bosons, it should have formed compact gravitating Bose-Einstein condensates (BEC) such as boson stars. Boson stars were introduced by Kaup (1968) and Ruffini \& Bonazzola (1969) in the 1960s. Early works on boson stars (Thirring 1983; Breit et al. 1984; Takasugi \& Yoshimura 1984; van der Bij \& Gleiser 1987) were motivated by the axion field that was proposed as a possible solution to the strong $\mathrm{CP}$ problem in QCD. For particles with mass $m \sim 1 \mathrm{GeV} / \mathrm{c}^{2}$, the maximum mass of a boson star, called the Kaup mass, is much lower than the solar mass $\left(M_{\text {Kaup }} \sim 10^{-19} M_{\odot}\right.$ !), so that these mini boson stars, such as axion black holes, are not very astrophysically relevant. They could play a role, however, if they exist in the universe in abundance or if the axion mass is extraordinary low (Schunck \& Mielke 2003), leading to macroscopic objects with a mass $M_{\text {Kaup }}$ comparable to the mass of the sun (or even 
higher). For example, axionic boson stars could account for the mass of MACHOs (between 0.3 and $0.8 M_{\odot}$ ) if the axions have a mass $m \sim 10^{-10} \mathrm{eV} / c^{2}$ (Mielke \& Schunck 2000). Some authors (Baldeschi et al. 1983; Sin 1994; Schunck 1998; Matos \& Guzmán 1999; Hu et al. 2000; Arbey et al. 2001; Silverman \& Mallett 2002) have also proposed that dark matter halos could be giant systems of "Bose liquid", but in that case the mass of the bosons must be extremely low $\left(m \sim 10^{-24} \mathrm{eV} / c^{2}\right)$ to yield masses consistent with the mass of galactic halos. Such an ultralight scalar field is called "fuzzy cold dark matter" (FCDM) by $\mathrm{Hu}$ et al. (2000), who discuss its overall cosmological behavior. On the other hand, Colpi et al. (1986) have shown that if the bosons have a self-interaction, then the mass of the boson stars can increase considerably, even for a small self-interaction. For $m \sim 1 \mathrm{GeV} / \mathrm{c}^{2}$ it approaches the solar mass so that dark matter could be made of numerous boson stars. On the other hand, for $m \sim 1 \mathrm{eV} / \mathrm{c}^{2}$, the mass of the boson stars approaches the mass of the galactic halos. Therefore, some authors (Lee \& Koh 1996; Peebles 2000; Goodman 2000; Arbey et al. 2003; Böhmer \& Harko 2007; Fukuyama et al. 2008) have proposed that dark matter halos themselves could be in the form of gigantic selfgravitating BECs with short-range interactions described by a single wave function $\psi(\boldsymbol{r}, t)$. In the Newtonian limit, which is relevant on the galactic scale, the evolution of this wave function is governed by the Gross-Pitaevskii-Poisson (GPP) system (Böhmer \& Harko 2007). With the Madelung (1927) transformation, the GP equation turns out to be equivalent to hydrodynamic (Euler) equations involving an isotropic classical pressure due to short-range interactions (scattering) and an anisotropic quantum pressure (or a quantum potential) arising from the Heisenberg uncertainty principle. For a standard BEC with quartic selfinteraction, the equation of state is that of a polytrope with index $n=1$. On large scales, scattering and quantum effects are negligible, and one recovers the classical hydrodynamic equations of cold dark matter (CDM) models that are remarkably successful in explaining the large-scale structure of the universe (Peebles \& Ratra 2003). On small scales, gravitational collapse is prevented by the (repulsive) scattering or by the uncertainty principle. This may be a way to solve the problems of the CDM model, such as the cusp and the missing satellite problems (Hu et al. 2000).

To this day, the BEC model has two main virtues. (i) It can reproduce the rotation curves of several low surface brightness (LSB) galaxies (Sin 1994; Lee \& Koh 1996; Schunck 1998; Guzmán \& Matos 2000; Arbey et al. 2003; Böhmer \& Harko 2007) and account for their almost flat behavior at long distances, and (ii) the quantum or scattering pressure can stabilize the system against gravitational collapse and lead to dark matter halos with flat central density profiles (Hu et al. 2000; Matos \& Ureña-López 2001; Bernal et al. 2008). This is interesting because there is strong observational evidence that the distribution of dark matter presents a nearly constant density core (Burkert 1995). For example, in the case of LSB galaxies and dwarf spiral galaxies, the content of baryons is small and these objects are dominated by dark matter. The Hi nearby galaxy survey (THINGS) of dwarf galaxies (Walter et al. 2008) has revealed discrepancies between observations and simulations done in the framework of the $\Lambda$ CDM model. In particular, the logarithmic inner slopes of the density profiles are on the order -0.2 (Oh et al. 2011), as for LSB galaxies (de Blok et al. 2003), instead of the -1 predicted by CDM simulations (Navarro et al. 1996). Therefore, the observations seem to favor flat density profiles over cuspy profiles. This is consistent with the BEC model, as shown in the recent paper of Harko (2011b). At the same time, this explains why the abundance of satellite galaxies predicted by CDM simulations is not observed around the Milky Way (Klypin et al. 1999). It is difficult to presume the relevance of the BEC model to account for all astrophysical observations, but there is a growing accumulation of recent material indicating that this model may be viable. In any case, it deserves to be studied in greater detail at the physical and astrophysical levels because it combines fundamental elements of gravity, quantum mechanics, and fluid mechanics.

In our previous papers (Chavanis 2011a; Chavanis \& Delfini 2011), we performed an exhaustive study of the equilibrium configurations of a Newtonian self-gravitating BEC with shortrange interactions. For a given value of the scattering length, we obtained the mass-radius relation $M(R)$ connecting the noninteracting case (corresponding to low masses) studied by Ruffini \& Bonazzola (1969) to the Thomas-Fermi (TF) limit (corresponding to large masses) investigated by Böhmer \& Harko (2007). We also considered the case of attractive self-interaction. This corresponds to a negative scattering length $\left(a_{\mathrm{s}}<0\right)$ yielding a negative pressure. In that case, we found there is a maximum mass $M_{\max }=1.012 \hbar / \sqrt{\left|a_{\mathrm{s}}\right| G m}$ above which the system becomes unstable. It turns out that this mass is ridiculously low (being possibly as low as the Planck mass $M_{\mathrm{P}}=2.1810^{-8} \mathrm{~kg}$ !), meaning that a self-gravitating BEC with attractive short-range interactions is extremely unstable (except if the mass of the bosons is extraordinarily low, on the order of $m \sim 10^{-22} \mathrm{eV} / c^{2}$, like in $\mathrm{Hu}$ et al. 2000). We proposed that the scattering length could be negative in the early universe and that it could help form structures $^{1}$. Then, the scattering length could become positive and help stabilize the structures against gravitational collapse. Of course, the mechanism by which the scattering length changes sign (due, for example, to a change in density) remains to be established so that this idea is highly speculative. We note that some atoms in terrestrial BEC experiments are reported to have negative scattering lengths (Dalfovo et al. 1999). Therefore, the possibility of negative pressure for a BEC can be contemplated. Furthermore, it has been experimentally demonstrated that, under certain conditions, it is possible to manipulate the sign and value of the scattering length (Fedichev et al. 1996). The extension of these ideas to cosmology remains an important problem that we shall not discuss further here. We assume that the dark matter in the universe is a BEC with repulsive $\left(a_{\mathrm{s}}>0\right)$ or attractive $\left(a_{\mathrm{S}}<0\right)$ self-interaction and theoretically explore the consequences of this hypothesis.

If dark matter halos are BECs, they have probably formed by Jeans instability. The gravitational instability of a scalar field equivalent to a BEC was considered by Khlopov et al. (1985); Bianchi et al. (1990); Hu et al. (2000); and Sikivie \& Yang (2009). However, these authors all started their analysis from relativistic field equations and did not take the self-interaction of the particles into account. In our previous paper (Chavanis 2011a), we studied the Jeans instability of a Newtonian selfgravitating BEC with short-range interactions described by the Gross-Pitaevskii-Poisson system in a static universe. We considered both attractive and repulsive self-interaction and found that when the scattering length is negative the growth rate of the instability increases with respect to the case where the scattering length is positive or zero. The next step is to study the Jeans instability of a BEC in an expanding universe. This is the topic of the present paper.

\footnotetext{
1 This is a potentially interesting idea because, in an expanding universe, the condensation process is often regarded as too slow to account for the formation of structures (Bonnor 1957; Harrison 1967). An attractive self-interaction could substantially enhance the instability.
} 
In the first part of the paper (Sects. 2-7), we neglect relativistic effects and use the equations of Newtonian cosmology introduced by Milne (1934) and McCrea \& Milne (1934). We study the linear development of perturbations of a self-gravitating BEC with short-range interactions in an expanding Einstein-de Sitter (EdS) universe. In the TF approximation, the equation of state of a BEC with quartic self-interaction is that of a polytrope of index $\gamma=2(n=1)$. In the noninteracting case, we find that the BEC behaves similarly to a polytrope of index $\gamma=5 / 3(n=3 / 2)$ but with a quantum mechanically modified Jeans length. In the two cases, the equation for the density contrast can be analytically solved in terms of Bessel functions.

In the second part of the paper (Sect. 8), we take special relativistic effects into account and use the equations of Newtonian cosmology with pressure introduced by McCrea (1951). In that case, the evolution of the cosmic fluid depends on the equation of state. We consider BEC dark matter with equation of state $p=k \rho^{2} c^{2}$ and add the contribution of radiation, baryons, and dark energy (cosmological constant). For BEC dark matter with repulsive self-interaction $k>0$ (positive pressure), the scale factor increases more rapidly than in the standard $\Lambda$ CDM model where dark matter is pressureless $(k=0)$, while it increases less rapidly for BEC dark matter with attractive self-interaction $k<0$ (negative pressure). We study the linear development of the perturbations in these two cases and show that the perturbations grow faster in BEC dark matter than in pressureless dark matter.

In the third part of the paper (Sect. 9), we consider a "dark fluid" with a generalized equation of state $p=\left(\alpha \rho+k \rho^{2}\right) c^{2}$ having a component $p=k \rho^{2} c^{2}$ similar to BEC dark matter and a component $p=\alpha \rho c^{2}$ mimicking the effect of the cosmological constant (dark energy). We find optimal parameters that give good agreement with the standard $\Lambda \mathrm{CDM}$ model. Therefore, this "mixed" equation of state could be an alternative to the cosmological constant.

While our series of papers on this subject was being written, Harko (2011a) published a paper where he also considered the formation of structures in an expanding universe made of BEC dark matter. Our independent contribution is complementary to Harko's work and confirms his main results. In addition, we address the following issues: (i) we study the effect of the quantum pressure in the first part of the paper; (ii) we use different relativistic hydrodynamic equations to model the cosmic fluid in the second part of the paper; (iii) we study a generalized equation of state in the third part of the paper, and (iv) we consider both positive and negative scattering lengths throughout the paper. The papers of Böhmer \& Harko (2007) and Harko (2011a) show that a cosmic BEC can be an interesting model for the dark matter of the universe. Our series of papers, which develop this idea, go in the same direction.

Finally, in a different perspective, Widrow \& Kaiser (1993) have proposed to describe a classical collisionless selfgravitating system by the Schrödinger-Poisson system. In this approach, the constant $\hbar$ is not the Planck constant, but rather an adjustable parameter that controls the spatial resolution $\lambda_{\mathrm{deB}}$ through a de Broglie relation $\lambda_{\mathrm{deB}}=\hbar / m v$. They argue that when $\hbar \rightarrow 0$, the Vlasov-Poisson system is recovered and that a finite value of $\hbar$ provides a small-scale regularization of the dynamics. In that case, the Schrödinger-Poisson system has nothing to do with quantum mechanics since it aims at describing the evolution of classical collisionless matter under the influence of gravity (in static or expanding universes). Still, from a mathematical point of view, these equations are equivalent to those describing selfgravitating BECs without self-interaction. Therefore, the results of the first part of our paper can have application in that context, independently of quantum mechanics.

\section{The Gross-Pitaevskii-Poisson system}

Following Böhmer \& Harko (2007), we assume that dark matter is a BEC. A self-gravitating BEC with short-range interactions is described by the Gross-Pitaevskii-Poisson system

$$
\begin{aligned}
i \hbar \frac{\partial \psi}{\partial t} & =-\frac{\hbar^{2}}{2 m} \Delta \psi+m(\Phi+h(\rho)) \psi \\
\Delta \Phi & =4 \pi G N m|\psi|^{2}-\Lambda
\end{aligned}
$$

where $\rho=N m|\psi|^{2}$ is the density, $\Phi$ the gravitational potential, and $h(\rho)=\int u_{\mathrm{SR}}\left(\boldsymbol{r}-\boldsymbol{r}^{\prime}\right) \rho\left(\boldsymbol{r}^{\prime}, t\right) \mathrm{d} \boldsymbol{r}^{\prime}$ an effective potential that takes small-scale interactions into account. For the sake of generality, we have included the cosmological constant $\Lambda$ in the Poisson equation ${ }^{2}$. We write the wave function in the form $\psi(\boldsymbol{r}, t)=A(\boldsymbol{r}, t) \mathrm{e}^{\mathrm{i} S(\boldsymbol{r}, t) / \hbar}$ where $A$ and $S$ are real, and make the Madelung (1927) transformation

$\rho=N m|\psi|^{2}=N m A^{2}, \quad \boldsymbol{u}=\frac{1}{m} \nabla S$,

where $\rho(\boldsymbol{r}, t)$ is the density field and $\boldsymbol{u}(\boldsymbol{r}, t)$ the velocity field. We note that the flow is irrotational since $\nabla \times \boldsymbol{u}=\mathbf{0}$. With this transformation, it can be shown that the Gross-Pitaevskii Eq. (1) is equivalent to the barotropic Euler equations with an additional term $Q$ called the quantum potential (or quantum pressure) arising from the Heisenberg uncertainty principle. Indeed, one obtains the set of equations

$$
\begin{aligned}
& \frac{\partial \rho}{\partial t}+\nabla \cdot(\rho \boldsymbol{u})=0, \\
& \frac{\partial \boldsymbol{u}}{\partial t}+(\boldsymbol{u} \cdot \nabla) \boldsymbol{u}=-\frac{1}{\rho} \nabla p-\nabla \Phi-\frac{1}{m} \nabla Q, \\
& \Delta \Phi=4 \pi G \rho-\Lambda,
\end{aligned}
$$

with

$Q=-\frac{\hbar^{2}}{2 m} \frac{\Delta \sqrt{\rho}}{\sqrt{\rho}}$

The pressure $p(\rho)$ is determined by the effective potential $h(\rho)$, playing the role of an enthalpy (Chavanis 2011a), through the relation $p^{\prime}(\rho)=\rho h^{\prime}(\rho)$. This yields $p(\rho)=\rho h(\rho)-H(\rho)$, where $H$ is a primitive of $h$. For a contact pair interaction $u_{\mathrm{SR}}\left(\boldsymbol{r}-\boldsymbol{r}^{\prime}\right)=$ $g \delta\left(\boldsymbol{r}-\boldsymbol{r}^{\prime}\right)$, the effective potential $h(\rho)=g \rho$ where $g=4 \pi a_{\mathrm{s}} \hbar^{2} / \mathrm{m}^{3}$ is the pseudo-potential and $a_{\mathrm{s}}$ is the s-scattering length (Dalfovo et al. 1999). For the sake of generality, we allow $a_{\mathrm{S}}$ to be positive or negative. The corresponding equation of state is

$p=\frac{2 \pi a_{\mathrm{s}} \hbar^{2}}{m^{3}} \rho^{2}$.

It corresponds to a polytropic equation of state of the form

$p=K \rho^{\gamma}, \quad \gamma=1+\frac{1}{n}$

2 We may note that the cosmological constant $\Lambda$ plays the same role as a global rotation $\Omega$ of the universe if $\Phi$ is interpreted as an effective gravitational potential $\Phi_{\text {eff }}=\Phi-\frac{1}{2}(\boldsymbol{\Omega} \times \boldsymbol{r})^{2}$ in the rotating frame. Indeed, the Poisson equation becomes $\Delta \Phi_{\text {eff }}=4 \pi G \rho-2 \Omega^{2}$, which allows the identification $\Lambda=2 \Omega^{2}$. 
with polytropic index $n=1$ (i.e. $\gamma=2$ ) and polytropic constant $K=2 \pi a_{\mathrm{s}} \hbar^{2} / m^{3}$.

The effective potential corresponding to the polytropic equation of state (9) is

$h(\rho)=\frac{K \gamma}{\gamma-1} \rho^{\gamma-1}$

This leads to a GP equation of the form

$\mathrm{i} \hbar \frac{\partial \psi}{\partial t}=-\frac{\hbar^{2}}{2 m} \Delta \psi+m\left(\Phi+\kappa|\psi|^{2 / n}\right) \psi$

where $\kappa=K(n+1)(N m)^{1 / n}$. This is the usual form of the GP equation considered in the literature (Sulem \& Sulem 1999). The standard BEC, with a quartic interaction $p \propto \rho^{2} \propto|\psi|^{4}$, corresponds to $n=1$. We may also recall that classical and ultra relativistic fermion stars are equivalent to polytropes with in$\operatorname{dex} n=3 / 2$ and $n=3$ respectively (Chandrasekhar 1939). The GPP system (11)-(2) with $n=3 / 2$ has been studied by Bilic et al. (2001) in relation to the formation of white dwarf stars by gravitational collapse. They show that the quantum pressure (Heisenberg) can regularize the dynamics on small scales.

For an isothermal equation of state

$p=\rho \frac{k_{\mathrm{B}} T_{\mathrm{eff}}}{m}$,

the effective potential is

$h(\rho)=\frac{k_{\mathrm{B}} T_{\mathrm{eff}}}{m} \ln \rho$,

and the GP equation reads as

$\mathrm{i} \hbar \frac{\partial \psi}{\partial t}=-\frac{\hbar^{2}}{2 m} \Delta \psi+\left(m \Phi+2 k_{\mathrm{B}} T_{\mathrm{eff}} \ln |\psi|\right) \psi$

Interestingly, a nonlinear Schrödinger equation with a logarithmic potential similar to Eq. (14) was introduced long ago by Bialynicki \& Mycielski (1976) as a possible generalization of the Schrödinger equation in quantum mechanics.

Remark: for a BEC at $T=0$, the pressure arising in the Euler Eq. (5) has a meaning different from the kinetic pressure of a normal fluid at finite temperature. It is due to the self-interaction of the particles encapsulated in the effective potential $h(\rho)$ and not to thermal motion (since $T=0$ ). As a result, the pressure can be negative (!) contrary to the kinetic pressure. This is the case, in particular, for a BEC described by the equation of state (8) when the scattering length $a_{\mathrm{s}}$ is negative. In terrestrial BEC experiments, some atoms like ${ }^{7} \mathrm{Li}$ have a negative scattering length (Fedichev et al. 1996). On the other hand, the constant $T_{\text {eff }}$ appearing in Eq. (12) is just an "effective" temperature since it arises from a particular form of self-interaction and has nothing to do with the kinetic temperature (which here is $T=0$ ). In particular, this effective temperature can be negative. In that respect, we note that linear equations of state $p=\alpha \rho c^{2}$ with $\alpha<0$ have been introduced heuristically to account for the accelerated expansion of the universe (see the review of Peebles \& Ratra 2003 and Sect. 9).

\section{Newtonian cosmology}

In this first part of the paper, we use the Newtonian cosmology introduced by Milne and McCrea \& Milne in 1934. The great advantage of the Newtonian treatment is its simplicity ${ }^{3}$. Furthermore, the equations are identical to those derived using the theory of general relativity, provided the pressure is negligible in comparison with the energy density $\rho c^{2}$ where $c$ is the speed of light (we go beyond this limitation in the second part of the paper). This makes this approach attractive. Furthermore, its simplicity allows us to introduce novel ingredients such as the quantum pressure that is derived from the classical Gross-Pitaevskii equation. The validity and limitation of Newtonian cosmology have been discussed by various authors such as Layzer (1954), McCrea (1955), Callan et al. (1965) and Harrison (1965).

We recall now the basics of Newtonian cosmology. We consider a spatially homogeneous solution of Eqs. (4)-(7) of the form

$\rho(\boldsymbol{r}, t)=\rho_{\mathrm{b}}(t), \quad \boldsymbol{u}(\boldsymbol{r}, t)=\frac{\dot{a}}{a} \boldsymbol{r}$,

where $a(t)$ is the scale factor and $H=\dot{a} / a$ is the Hubble "constant" (in fact a function of time). The Euler Eqs. (4)-(5) reduce to

$$
\begin{aligned}
& \frac{\mathrm{d} \rho_{\mathrm{b}}}{\mathrm{d} t}+3 \rho_{\mathrm{b}} \frac{\dot{a}}{a}=0, \\
& \frac{\ddot{a}}{a} \boldsymbol{r}=-\nabla \Phi_{\mathrm{b}} .
\end{aligned}
$$

The pressure $p$ and the quantum potential $Q$ do not appear in the theory of the homogeneous model since they enter Eq. (5) only through their gradients. Equation (16) leads to the relation

$\rho_{\mathrm{b}} a^{3} \sim 1$,

which corresponds to the conservation of mass. Taking the divergence of Eq. (17) and using the Poisson Eq. (6), we obtain the cosmological equation

$\frac{\mathrm{d}^{2} a}{\mathrm{~d} t^{2}}=-\frac{4}{3} \pi G \rho_{\mathrm{b}} a+\frac{\Lambda}{3} a$.

Using Eq. (18), its first integral is

$\left(\frac{\mathrm{d} a}{\mathrm{~d} t}\right)^{2}=\frac{1}{3}\left(8 \pi G \rho_{\mathrm{b}}+\Lambda\right) a^{2}-\kappa$,

where $\kappa$ is a constant of integration. Equations (18)-(20) are the Newtonian equations of an isotropic and homogeneous universe. They coincide with the equations derived by Friedmann (1922, 1924) for $\Lambda=0$ and $\kappa= \pm 1$ and by Einstein \& de Sitter (1932) for $\Lambda=0$ and $\kappa=0$ from the theory of general relativity when the pressure is much lower than the energy density $\rho c^{2}$. In that case, $\kappa$ is the curvature constant and space is flat $(\kappa=0)$, elliptical $(\kappa=1)$, or hyperbolic $(\kappa=-1)^{4}$.

The Einstein (1917) static universe corresponds to

$a=1, \quad \rho_{\mathrm{b}}=\frac{\Lambda}{4 \pi G}, \quad \kappa=\Lambda$.

${ }^{3}$ It is surprising to realize that Newtonian cosmology was developed after, and was influenced by, the cosmological models based on Einstein's theory of general relativity. It could have been developed much earlier. As Milne writes: "It seems to have escaped previous notice that whereas the theory of the expanding universe is generally held to be one of the fruits of the theory of relativity, actually all the phenomena observable at present could have been predicted by the founders of mathematical hydrodynamics in the eighteenth century, or even by Newton himself".

${ }^{4}$ The constant $\kappa$ in Eq. (20) can be set to unity by a suitable normalization of the parameters. 
However, this universe is unstable against perturbations in $a$ (Eddington 1930, Harrison 1967). The Einstein-de Sitter (EdS) universe corresponds to $\Lambda=0$ and $\kappa=0$. In that case, Eqs. (19) and (20) reduce to

$\ddot{a}=-\frac{4}{3} \pi G \rho_{\mathrm{b}} a, \quad \dot{a}^{2}=\frac{8}{3} \pi G \rho_{\mathrm{b}} a^{2}$.

This yields

$a \propto t^{2 / 3}, \quad H=\frac{\dot{a}}{a}=\frac{2}{3 t}, \quad \rho_{\mathrm{b}}=\frac{1}{6 \pi G t^{2}}$.

Both inflationary theory (Guth 1981) and observations favor a flat universe $(\kappa=0)$. In that case, Eq. (20) can be written $H^{2}=$ $\frac{8}{3} \pi G\left(\rho_{\mathrm{b}}+\rho_{\Lambda}\right)$, where $\rho_{\Lambda}=\Lambda / 8 \pi G$ is the dark energy density, and it gives a relationship between Hubble's constant and the total density of the universe. With the present-day value of the Hubble constant $H_{0}=2.273 \times 10^{-18} \mathrm{~s}^{-1}$, the Einstein-de Sitter model $\left(\kappa=\Lambda=0, p \ll \rho c^{2}\right)$ leads to an age of the universe $t_{0}=2 / 3 H_{0} \sim 9.3$ billion years, while the $\Lambda \mathrm{CDM}$ model, which is the standard model of cosmology, predicts 13.75 billion years. The effect of dark energy is considered in Sects. 8.4 and 9.

Remark: we can obtain Eqs. (18)-(20) directly if we use the "naive" picture that the universe is a uniform sphere of radius $a(t)$, density $\rho_{\mathrm{b}}(t)$, and mass $M$. The conservation of mass $M=\frac{4}{3} \pi \rho_{\mathrm{b}} a^{3}$ leads to Eq. (18). On the other hand, applying the Gauss theorem at the border of the sphere, Newton's equation can be written $\mathrm{d}^{2} a / \mathrm{d} t^{2}=-G M / a^{2}+\Lambda a / 3$ leading to Eq. (19). This is the equation of motion of a fictive particle of position $a$ in a potential $V(a)=-G M / a-\Lambda a^{2} / 6$. Its first integral is given by Eq. (20) where $E=-\kappa / 2$ can be regarded as the energy of the fictive particle. The case $E=0$ (EdS universe) corresponds to the situation where the velocity of the fictive particle is equal to the escape velocity. In this naive picture, $a$ may be interpreted as the "radius" of the universe. In fact, a better derivation (which does not assume a finite sphere) is to apply Newton's equation to an arbitrary fluid particle located in $\boldsymbol{r}$ and write $\mathrm{d}^{2} r / \mathrm{d} t^{2}=-G\left(4 \pi \rho_{\mathrm{b}} r^{3} / 3\right) / r^{2}+\Lambda r / 3$. Setting $\boldsymbol{r}=a(t) \boldsymbol{x}$ and dividing by $x$ yields Eq. (19).

\section{Quantum Euler-Poisson system in an expanding universe}

We now study the instability of the homogeneous background and the growth of perturbations that ultimately lead to the largescale structures of the universe. We work in the comoving frame (Peebles 1980). To that purpose, we set

$\boldsymbol{r}=a(t) \boldsymbol{x}, \quad \boldsymbol{u}=\frac{\dot{a}}{a} \boldsymbol{r}+\boldsymbol{v}$,

where $v$ is the peculiar velocity. For the moment, we allow arbitrary deviations from the background flow. We first write the Poisson Eq. (6) in the comoving frame. Integrating Eq. (17) and using Eq. (19), we find that the background gravitational potential is

$\Phi_{\mathrm{b}}=-\frac{1}{2} \frac{\ddot{a}}{a} r^{2}=-\frac{1}{2} \ddot{a} a x^{2}=\frac{2}{3} \pi G \rho_{\mathrm{b}}(t) r^{2}-\frac{\Lambda}{6} r^{2}$.

This result can also be obtained by integrating the Poisson equation for a homogeneous system or by using the Gauss theorem. If we introduce the new potential $\phi=\Phi-\Phi_{\mathrm{b}}$, i.e.

$\phi=\Phi+\frac{1}{2} \ddot{a} a x^{2}$, the Poisson Eq. (6) becomes

$\Delta \phi=4 \pi G a^{2}\left(\rho-\rho_{\mathrm{b}}\right)$,

where the Laplacian is taken with respect to $\boldsymbol{x}$. Now, following Peebles (1980) and taking the quantum pressure into account, we find that the hydrodynamic Eqs. (4) and (5) can be written in the comoving frame as

$$
\begin{aligned}
\frac{\partial \rho}{\partial t}+ & \frac{3 \dot{a}}{a} \rho+\frac{1}{a} \nabla \cdot(\rho \boldsymbol{v})=0, \\
\frac{\partial \boldsymbol{v}}{\partial t}+ & \frac{1}{a}(\boldsymbol{v} \cdot \nabla) \boldsymbol{v}+\frac{\dot{a}}{a} \boldsymbol{v}=-\frac{1}{\rho a} \nabla p-\frac{1}{a} \nabla \phi \\
& +\frac{\hbar^{2}}{2 m^{2} a^{3}} \nabla\left(\frac{\Delta \sqrt{\rho}}{\sqrt{\rho}}\right) .
\end{aligned}
$$

It is convenient to write the density in the form

$\rho=\rho_{\mathrm{b}}(t)[1+\delta(\boldsymbol{x}, t)]$,

where $\rho_{\mathrm{b}} \propto 1 / a^{3}$ and $\delta(\boldsymbol{x}, t)$ is the density contrast (Peebles 1980). Substituting Eq. (30) into Eqs. (27)-(29), we obtain the quantum barotropic Euler-Poisson system in an expanding universe

$$
\begin{aligned}
\frac{\partial \delta}{\partial t}+ & \frac{1}{a} \nabla \cdot((1+\delta) \boldsymbol{v})=0 \\
\frac{\partial \boldsymbol{v}}{\partial t}+ & \frac{1}{a}(\boldsymbol{v} \cdot \nabla) \boldsymbol{v}+\frac{\dot{a}}{a} \boldsymbol{v}=-\frac{1}{\rho a} \nabla p-\frac{1}{a} \nabla \phi \\
& +\frac{\hbar^{2}}{2 m^{2} a^{3}} \nabla\left(\frac{\Delta \sqrt{1+\delta}}{\sqrt{1+\delta}}\right), \\
\Delta \phi & =4 \pi G \rho_{\mathrm{b}} a^{2} \delta
\end{aligned}
$$

For $\hbar=p=0$, we recover the usual Euler equations of a cold gas $(T=0)$ in an expanding universe (Peebles 1980) ${ }^{5}$. For a barotropic equation of state $p=p(\rho)$, the Euler Eq. (32) can be rewritten

$$
\begin{aligned}
\frac{\partial \boldsymbol{v}}{\partial t}+\frac{1}{a}(\boldsymbol{v} \cdot \nabla) \boldsymbol{v}+\frac{\dot{a}}{a} \boldsymbol{v} & =-\frac{c_{\mathrm{s}}^{2}}{(1+\delta) a} \nabla \delta-\frac{1}{a} \nabla \phi \\
& +\frac{\hbar^{2}}{2 m^{2} a^{3}} \nabla\left(\frac{\Delta \sqrt{1+\delta}}{\sqrt{1+\delta}}\right),
\end{aligned}
$$

where $c_{\mathrm{s}}^{2}=p^{\prime}(\rho)=p^{\prime}\left(\rho_{\mathrm{b}}(1+\delta)\right)$ is the square of the velocity of sound in the evolving system. It generically depends on position and time. For an isothermal equation of state, $c_{\mathrm{s}}^{2}=k_{\mathrm{B}} T / m$ is a constant and for a polytropic equation of state $c_{\mathrm{s}}^{2}=K \gamma \rho^{\gamma-1}$. For a standard BEC with a quartic self-interaction, described by the equation of state (8), the square of the velocity of sound is

$c_{\mathrm{s}}^{2}=\frac{4 \pi a_{\mathrm{s}} \hbar^{2} \rho}{m^{3}}$,

${ }^{5}$ If the universe is a classical collisionless fluid, the evolution of this fluid is fundamentally described by the Vlasov equation (Gilbert 1966). In that case, the hydrodynamic equations without pressure $(\hbar=p=0)$ are based on some approximations (Peebles 1980). They assume the existence of a single fluid velocity at every spatial position, and they cease to be valid after the first time of crossing where multi streaming generates a range of particle velocities through a given point (Vergassola et al. 1994). Alternatively, if dark matter is a BEC, the hydrodynamic Eqs. (31)-(33) are rigorously equivalent to the GPP system for all times. 
and we obtain the system of equations

$$
\begin{aligned}
& \frac{\partial \delta}{\partial t}+\frac{1}{a} \nabla \cdot((1+\delta) \boldsymbol{v})=0, \\
& \frac{\partial \boldsymbol{v}}{\partial t}+\frac{1}{a}(\boldsymbol{v} \cdot \nabla) \boldsymbol{v}+\frac{\dot{a}}{a} \boldsymbol{v}=-\frac{4 \pi a_{\mathrm{s}} \hbar^{2} \rho_{\mathrm{b}}}{m^{3} a} \nabla \delta \\
& -\frac{1}{a} \nabla \phi+\frac{\hbar^{2}}{2 m^{2} a^{3}} \nabla\left(\frac{\Delta \sqrt{1+\delta}}{\sqrt{1+\delta}}\right), \\
& \Delta \phi=4 \pi G \rho_{\mathrm{b}} a^{2} \delta .
\end{aligned}
$$

\section{Linearized equations}

Of course, $\delta=\phi=0$ and $\boldsymbol{v}=\mathbf{0}$ is a solution of the quantum Euler-Poisson system (31)-(33) in the comoving frame, corresponding to the pure background flow (15). However, this solution may be unstable to some perturbations. If we consider small perturbations $\delta \ll 1, \phi \ll 1,|\boldsymbol{v}| \ll 1$ and linearize the foregoing equations we obtain

$$
\begin{aligned}
& \frac{\partial \delta}{\partial t}+\frac{1}{a} \nabla \cdot \boldsymbol{v}=0, \\
& \frac{\partial \boldsymbol{v}}{\partial t}+\frac{\dot{a}}{a} \boldsymbol{v}=-\frac{1}{a} c_{\mathrm{s}}^{2} \nabla \delta-\frac{1}{a} \nabla \phi+\frac{\hbar^{2}}{4 m^{2} a^{3}} \nabla(\Delta \delta), \\
& \Delta \phi=4 \pi G \rho_{\mathrm{b}} \delta a^{2},
\end{aligned}
$$

where $c_{\mathrm{s}}^{2}=p^{\prime}\left(\rho_{\mathrm{b}}(t)\right)$ now denotes the square of the velocity of sound in the homogeneous background flow. It is just a function of time. Taking the time derivative of Eq. (39) multiplied by $a$, the divergence of Eq. (40), and using Eq. (41), these equations can be combined into a single equation governing the evolution of the density contrast

$$
\frac{\partial^{2} \delta}{\partial t^{2}}+2 \frac{\dot{a}}{a} \frac{\partial \delta}{\partial t}=\frac{c_{\mathrm{s}}^{2}}{a^{2}} \Delta \delta+4 \pi G \rho_{\mathrm{b}} \delta-\frac{\hbar^{2}}{4 m^{2} a^{4}} \Delta^{2} \delta .
$$

For $\hbar=0$, we recover the equation first derived by Bonnor (1957). In his case, the pressure $p$ is a kinetic pressure. Expanding the solution in Fourier modes of the form $\delta(\boldsymbol{x}, t)=$ $\delta_{k}(t) \mathrm{e}^{i \boldsymbol{k} \cdot \boldsymbol{x}}$, we obtain

$$
\ddot{\delta}+2 \frac{\dot{a}}{a} \dot{\delta}+\left(\frac{\hbar^{2} k^{4}}{4 m^{2} a^{4}}+\frac{c_{\mathrm{s}}^{2} k^{2}}{a^{2}}-4 \pi G \rho_{\mathrm{b}}\right) \delta=0,
$$

where, for brevity, we have noted $\delta(t)$ for $\delta_{k}(t)$. For a standard $\mathrm{BEC}$, using $c_{\mathrm{s}}^{2}=4 \pi a_{\mathrm{s}} \hbar^{2} \rho_{\mathrm{b}} / m^{3}$, the foregoing equation can be rewritten

$\ddot{\delta}+2 \frac{\dot{a}}{a} \dot{\delta}+\left(\frac{\hbar^{2} k^{4}}{4 m^{2} a^{4}}+\frac{4 \pi a_{\mathrm{s}} \hbar^{2} \rho_{\mathrm{b}} k^{2}}{m^{3} a^{2}}-4 \pi G \rho_{\mathrm{b}}\right) \delta=0$.

\section{Quantum Jeans length}

\subsection{Expanding universe}

In the noninteracting case $a_{\mathrm{s}}=c_{\mathrm{s}}=0$, the equation for the density contrast reduces to

$\ddot{\delta}+2 \frac{\dot{a}}{a} \dot{\delta}+\left(\frac{\hbar^{2} k^{4}}{4 m^{2} a^{4}}-4 \pi G \rho_{\mathrm{b}}\right) \delta=0$.

From this relation, we can define a time-dependent quantum Jeans wavenumber

$k_{Q}=\left(\frac{16 \pi G \rho_{\mathrm{b}} m^{2} a^{4}}{\hbar^{2}}\right)^{1 / 4}$.
The proper quantum Jeans wavenumber obtained by writing $\delta \propto \mathrm{e}^{i \boldsymbol{k}_{*} \cdot \boldsymbol{r}}$ is $k_{Q}^{*}=\left(16 \pi G \rho_{\mathrm{b}} m^{2} / \hbar^{2}\right)^{1 / 4}$, but the comoving quantum Jeans wavenumber $k_{Q}$ is better suited to our problem. Recalling Eq. (18), we can write $k_{Q}=\kappa_{Q} a^{1 / 4}$ where $\kappa_{Q}=$ $\left(16 \pi G \rho_{\mathrm{b}} a^{3} m^{2} / \hbar^{2}\right)^{1 / 4}$ is a constant. The quantum Jeans length $\lambda_{Q}=2 \pi / k_{Q}$ decreases with time like $a^{-1 / 4}$ so that, in the comoving frame, the system becomes unstable on smaller and smaller scales as the universe expands (in contrast, the proper quantum Jeans scale increases with time as $\left.a^{3 / 4}\right)$. In the cold, nonquantum, universe ( $a_{\mathrm{s}}=c_{\mathrm{s}}=\hbar=0$ ), there is no Jeans length: all the scales are unstable. Therefore, quantum effects can stabilize the system on small scales, on the order of $\lambda_{Q}$, and avoid density cusps (Hu et al. 2000).

In the TF limit where the quantum potential can be neglected, the equation for the density contrast reduces to

$\ddot{\delta}+2 \frac{\dot{a}}{a} \dot{\delta}+\left(\frac{c_{\mathrm{s}}^{2} k^{2}}{a^{2}}-4 \pi G \rho_{\mathrm{b}}\right) \delta=0$.

From this relation, we can define a time-dependent classical Jeans wavenumber

$k_{\mathrm{J}}=\left(\frac{4 \pi G \rho_{\mathrm{b}} a^{2}}{c_{\mathrm{s}}^{2}}\right)^{1 / 2}$.

The proper Jeans wavenumber is $k_{\mathrm{J}}^{*}=\left(4 \pi G \rho_{\mathrm{b}} / c_{\mathrm{s}}^{2}\right)^{1 / 2}$. The evolution of $k_{\mathrm{J}}(t)$ depends on the equation of state. For an isothermal equation of state for which $c_{\mathrm{s}}^{2}=k_{\mathrm{B}} T / m$, recalling Eq. (18), we can write $k_{\mathrm{J}}=\kappa_{\mathrm{J}} a^{-1 / 2}$ where $\kappa_{\mathrm{J}}=\left(4 \pi G \rho_{\mathrm{b}} a^{3} m / k_{\mathrm{B}} T\right)^{1 / 2}$ is a constant. The classical Jeans length $\lambda_{\mathrm{J}}=2 \pi / k_{\mathrm{J}}$ increases with time like $a^{1 / 2}$ so that, in the comoving frame, the system becomes unstable on larger and larger scales as the universe expands. For a polytropic equation of state for which $c_{\mathrm{s}}^{2}=K \gamma \rho_{\mathrm{b}}^{\gamma-1}$, we can write $k_{\mathrm{J}}=\kappa_{\mathrm{J}} a^{(3 \gamma-4) / 2}$ where $\kappa_{\mathrm{J}}=\left[4 \pi G\left(\rho_{\mathrm{b}} a^{3}\right)^{2-\gamma} / K \gamma\right]^{1 / 2}$ is a constant. The classical Jeans wavelength behaves like $\lambda_{\mathrm{J}} \propto a^{(4-3 \gamma) / 2}$. For $\gamma<4 / 3$, the Jeans wavelength grows with time and for $\gamma>4 / 3$, it decreases with time. For $\gamma=4 / 3$, the Jeans wavenumber is constant in time, i.e. $k_{\mathrm{J}}=\kappa_{\mathrm{J}}$. Finally, for a standard BEC, $c_{\mathrm{s}}^{2}=4 \pi a_{\mathrm{s}} \hbar^{2} \rho_{\mathrm{b}} / \mathrm{m}^{3}$, the Jeans wavenumber can be written

$k_{\mathrm{J}}=\left(\frac{G m^{3} a^{2}}{a_{\mathrm{s}} \hbar^{2}}\right)^{1 / 2}$

It is independent of the density. Since $k_{\mathrm{J}}=\kappa_{\mathrm{J}} a$ with $\kappa_{\mathrm{J}}=$ $\left(G m^{3} / a_{\mathrm{S}} \hbar^{2}\right)^{1 / 2}$, the Jeans length decreases like $\lambda_{\mathrm{J}} \propto 1 / a$.

Remark: the study of the Jeans instability in an expanding universe exhibits a critical value of the polytropic index $\gamma_{\text {crit }}=4 / 3$ (i.e. $n_{\text {crit }}=3$ ). It is interesting to note that the same critical index arises when one studies the dynamical stability of spatially inhomogeneous polytropic spheres with respect to the barotropic Euler-Poisson system. It has been established that a polytropic star is stable if $\gamma \geq 4 / 3$ and unstable otherwise (Binney \& Tremaine 1987). Surprisingly, the same index appears in the stability analysis of a spatially homogeneous polytropic gas in an expanding universe. The static study of Jeans (see the following section) does not directly exhibit a critical value of the polytropic index 6 .

6 The critical value $\gamma_{\text {crit }}=4 / 3$ only appears when one introduces the Jeans mass $M_{\mathrm{J}} \sim \rho \lambda_{\mathrm{J}}^{3} \propto \rho^{(3 \gamma-4) / 2}$, but it does not play any particular role in the stability analysis of the homogeneous fluid. 


\subsection{Static universe}

If we assume that Eq. (43) remains valid in a static universe, and take $a=1$, we get

$\ddot{\delta}+\left(\frac{\hbar^{2} k^{4}}{4 m^{2}}+c_{\mathrm{s}}^{2} k^{2}-4 \pi G \rho_{\mathrm{b}}\right) \delta=0$.

Writing the perturbation in the form $\delta(t) \propto \mathrm{e}^{-\mathrm{i} \omega t}$, we obtain the dispersion relation

$\omega^{2}=\frac{\hbar^{2} k^{4}}{4 m^{2}}+c_{\mathrm{s}}^{2} k^{2}-4 \pi G \rho_{\mathrm{b}}$.

This dispersion relation has been studied in our previous paper (Chavanis 2011a). The generalized Jeans wavenumber is given by

$k_{\mathrm{c}}^{2}=\frac{2 m^{2}}{\hbar^{2}}\left[\sqrt{c_{\mathrm{s}}^{4}+\frac{4 \pi G \hbar^{2} \rho_{\mathrm{b}}}{m^{2}}}-c_{\mathrm{s}}^{2}\right]$.

In the noninteracting case $\left(a_{\mathrm{s}}=c_{\mathrm{s}}=0\right)$, we recover the quantum Jeans wavenumber (46) with $a=1$, and in the TF limit, we recover the classical Jeans wavenumber (48) with $a=1$. The dispersion relation can be written $\omega^{2} / 4 \pi G \rho_{\mathrm{b}}=k^{4} / k_{Q}^{4}+k^{2} / k_{\mathrm{J}}^{2}-1$, and the generalized Jeans wavenumber $k_{\mathrm{c}}^{2}=\left(k_{Q}^{4} / 2 k_{\mathrm{J}}^{2}\right)[ \pm(1+$ $\left.\left.4 k_{\mathrm{J}}^{4} / k_{Q}^{4}\right)^{1 / 2}-1\right]$ with + when $c_{\mathrm{s}}^{2} \geq 0$ and - when $c_{\mathrm{s}}^{2}<0$. For $\lambda<\lambda_{\mathrm{c}}, \omega$ is real and the perturbation oscillates with a pulsation $\omega$; for $\lambda>\lambda_{\mathrm{c}}, \omega$ is purely imaginary and the perturbation grows exponentially rapidly with a growth rate $\gamma=\sqrt{-\omega^{2}}$. The Jeans instability criterion is therefore $\lambda>\lambda_{\mathrm{c}}$ (i.e. $k<k_{\mathrm{c}}$ ). For $c_{\mathrm{s}}^{2} \geq 0$, the maximum growth rate corresponds to $k=0$ (infinite wavelengths) and is given by $\gamma=\sqrt{4 \pi G \rho_{\mathrm{b}}}$. On the other hand, considering a BEC with negative scattering length, for which $c_{\mathrm{s}}^{2}<0$, it is found (Chavanis 2011a) that the maximum growth rate corresponds to $k_{*}=\left(8 \pi\left|a_{\mathrm{s}}\right| \rho_{\mathrm{b}} / m\right)^{1 / 2}$ and is given by

$\gamma_{*}=\sqrt{\frac{16 \pi^{2} a_{\mathrm{s}}^{2} \hbar^{2} \rho_{\mathrm{b}}^{2}}{m^{4}}+4 \pi G \rho_{\mathrm{b}}}$.

Note that $k_{*}=\left(k_{Q}^{4} / 2\left|k_{\mathrm{J}}^{2}\right|\right)^{1 / 2}$ and $\gamma_{*}=\sqrt{4 \pi G \rho}\left(1+k_{Q}^{4} / 4 k_{\mathrm{J}}^{4}\right)^{1 / 2}$. For $\left|a_{\mathrm{s}}\right| \gg\left(G m^{4} / 4 \pi \hbar^{2} \rho_{\mathrm{b}}\right)^{1 / 2}$, we find that $\gamma_{*} \sim 4 \pi\left|a_{\mathrm{s}}\right| \hbar \rho_{\mathrm{b}} / m^{2} \gg$ $\sqrt{4 \pi G \rho_{\mathrm{b}}}$. Therefore, an attractive short-range interaction $\left(a_{\mathrm{s}}<\right.$ 0 ) increases the growth rate of the Jeans instability.

Jeans' classical analysis (Jeans 1902, 1929) suffers from the defect that, in general, there is no initial stationary state that is in a uniform nonrotating fluid. Therefore, using Eq. (50) in a static universe is referred to as the "Jeans swindle" . As noted by Bonnor (1957), the Jeans procedure is only valid in the static Einstein universe ${ }^{8}$. However, in this last case, the back-

\footnotetext{
7 Kiessling (2003) provides a vindication of the "Jeans swindle". He argues that, when considering an infinite and homogeneous distribution of matter, the Poisson equation must be modified so as to correctly define the gravitational force. He proposes to use a regularization of the form $\Delta \Phi-k_{0}^{2} \Phi=4 \pi G \rho$ where $k_{0}$ is an inverse screening length that ultimately tends to zero $\left(k_{0} \rightarrow 0\right)$, or a regularization of the form $\Delta \Phi=4 \pi G(\rho-\bar{\rho})$ where $\bar{\rho}$ is the mean density. In his point of view, this is not a swindle but just the right way to make the problem mathematically rigorous and have a well-defined gravitational force.

8 As is well known, Einstein (1917) introduced a cosmological constant in the equations of general relativity in order to recover a homogeneous static universe. He also considered, as a preamble of his paper, a modification of the classical Poisson equation in the form $\Delta \Phi-k_{0}^{2} \Phi=$ $4 \pi G \rho$ because he (incorrectly) believed that, in the Newtonian world model, the cosmological constant is equivalent to a screening length (see Spiegel 1998; Kiessling 2003 and Chavanis \& Delfini 2010 for historical details).
}

ground density of the universe is $\rho_{\mathrm{b}}=\Lambda / 4 \pi G$. The justification does not apply to a uniform mass of gas with a different density. Furthermore, the Einstein universe is strongly unstable. Therefore, this justification is not valid, and it is necessary to develop the Jeans instability analysis in an expanding universe.

Remark: Jeans analysis could be valid if the growth rate of the instability were much greater than the rate of the expansion of the universe. According to Eq. (22), the rate of expansion of the universe is $H=\dot{a} / a=(8 \pi G \rho / 3)^{1 / 2}$. On the other hand, when $c_{\mathrm{s}}^{2}>0$, the maximum growth rate of the Jeans instability is $\gamma=$ $\sqrt{4 \pi G \rho}$. They are exactly of the same order of magnitude, which leads to the usual conclusion that the Jeans instability analysis is not valid (see, e.g., Weinberg 1972). However, when $c_{\mathrm{s}}^{2}<0$, the maximum growth rate given by Eq. (53) can be much higher. In that case, the expansion of the universe could be ignored and the static Jeans instability analysis could be valid.

\section{Solution in the Einstein-de Sitter universe}

\subsection{The fundamental differential equation}

The evolution of the perturbations is more complicated to analyze in an expanding universe than in a static universe, and it turns out to be very different. For simplicity, we consider an Einstein-de Sitter universe. In that case, it is possible to obtain analytical solutions of the linearized Eq. (43) for the density contrast in some particular cases. Measuring the evolution in terms of $a$ instead of $t$ and using Eq. (22), Eq. (43) is transformed into $\frac{\mathrm{d}^{2} \delta}{\mathrm{d} a^{2}}+\frac{3}{2 a} \frac{\mathrm{d} \delta}{\mathrm{d} a}+\frac{3}{2 a^{2}}\left(\frac{\hbar^{2} k^{4}}{16 \pi G \rho_{\mathrm{b}} m^{2} a^{4}}+\frac{c_{\mathrm{s}}^{2} k^{2}}{4 \pi G \rho_{\mathrm{b}} a^{2}}-1\right) \delta=0$.

For $\hbar=c_{\mathrm{s}}=0$, we recover the case of a cold classical universe (dust) in which the pressure is zero and quantum effects are neglected. Equation (54) reduces to

$\frac{\mathrm{d}^{2} \delta}{\mathrm{d} a^{2}}+\frac{3}{2 a} \frac{\mathrm{d} \delta}{\mathrm{d} a}-\frac{3}{2 a^{2}} \delta=0$,

and its solutions are

$\delta_{+} \propto a, \quad \delta_{-} \propto a^{-3 / 2}$.

The growth of perturbations is algebraic in an expanding universe, while the "naive" Jeans instability analysis in a static universe predicts an exponential growth. Therefore, the results of the two analysis are very different. As mentioned long ago by Bonnor (1957) and others, this (slow) algebraic growth may be a problem for forming large-scale structures rapidly enough.

Assuming a polytropic equation of state $p=K \rho^{\gamma}$ for which $c_{\mathrm{s}}^{2}=K \gamma \rho_{\mathrm{b}}^{\gamma-1}$, and introducing the variables of Sect. 6.1, we can rewrite Eq. (54) in the form

$\frac{\mathrm{d}^{2} \delta}{\mathrm{d} a^{2}}+\frac{3}{2 a} \frac{\mathrm{d} \delta}{\mathrm{d} a}+\frac{3}{2 a^{2}}\left(\frac{k^{4}}{\kappa_{Q}^{4} a}+\frac{k^{2}}{\kappa_{\mathrm{J}}^{2} a^{3 \gamma-4}}-1\right) \delta=0$.

Making the change in variables $\delta(a)=f(a) / a$, we find that the differential equation for $f$ is

$\frac{\mathrm{d}^{2} f}{\mathrm{~d} a^{2}}-\frac{1}{2 a} \frac{\mathrm{d} f}{\mathrm{~d} a}+\frac{1}{a^{2}}\left(\frac{3 k^{4}}{2 \kappa_{Q}^{4} a}+\frac{3 k^{2}}{2 \kappa_{\mathrm{J}}^{2} a^{3 \gamma-4}}-1\right) f=0$.

We have not been able to find the general solution of this equation in terms of simple functions, so we consider particular cases. Some of the solutions presented below have already been discussed in the literature (see, e.g., Harrison 1967), but we give more details and put emphasis on the solutions describing selfgravitating BECs that were not discussed previously. 


\subsection{The TF approximation}

In the $\mathrm{TF}$ approximation where the quantum potential can be neglected, Eq. (58) reduces to

$\frac{\mathrm{d}^{2} f}{\mathrm{~d} a^{2}}-\frac{1}{2 a} \frac{\mathrm{d} f}{\mathrm{~d} a}+\frac{1}{a^{2}}\left(\frac{3 k^{2}}{2 \kappa_{\mathrm{J}}^{2} a^{3 \gamma-4}}-1\right) f=0$.

This is a particular case of the equation studied by Savedoff \& Vila (1962) in relation to the work of Bonnor $(1957)^{9}$. It can be solved in terms of Bessel functions (see Appendix A). For $\gamma \neq 4 / 3, f(a)$ is given by Eq. (A.7) and the evolution of the density contrast is

$\delta_{ \pm}(a) \propto \frac{1}{a^{1 / 4}} J_{ \pm \frac{5}{2(4-3 \gamma)}}\left(\frac{\sqrt{6}}{|4-3 \gamma|} \frac{k}{\kappa_{\mathrm{J}}} a^{\frac{4-3 \gamma}{2}}\right)$.

Using the asymptotic expansions of the Bessel functions (see Appendix A), we note that the density contrast is oscillating for $k \gg k_{\mathrm{J}}(a)$ and growing for $k \ll k_{\mathrm{J}}(a)$ (on the timescale over which these inequalities are fulfilled). Therefore, wavelengths that are longer than $\lambda_{\mathrm{J}}(a)$ behave like in a cold universe, whereas the perturbations have an oscillatory behavior for the short wavelengths. In this sense, the results are similar to those obtained with the classical Jeans analysis. They are, in fact, different because (i) the Jeans length varies with time; (ii) the oscillating solutions can be growing or decaying; and (iii) the growth is algebraic instead of exponential. The results depend more crucially on the value of the polytropic index $\gamma$ than on the Jeans length $\lambda_{\mathrm{J}}$. We must distinguish four cases. (i) $\gamma<4 / 3$ : for small $a$, the perturbations grow like in a cold gas $\left(\delta_{+} \sim a\right)$ and, for large $a$, they undergo damped oscillations (they decay like $1 / a^{(5-3 \gamma) / 4}$ ); (ii) $4 / 3<\gamma<5 / 3$ : for $a \rightarrow 0$ the perturbations diverge like $1 / a^{(5-3 \gamma) / 4}$ while oscillating, implying that we must start the stability analysis at $a_{i}>0$. For small $a \geq a_{i}$, the perturbations undergo damped oscillations (they decay like $1 / a^{(5-3 \gamma) / 4}$ ) and, for large $a$, they grow like in a cold gas $\left(\delta_{+} \sim a\right)$; (iii) $\gamma=5 / 3$ : for small $a$, the perturbations show pure oscillation and, for large $a$, they grow like in a cold gas $\left(\delta_{+} \sim a\right)$; (iv) $\gamma>5 / 3$ : for small $a$, the perturbations undergo growing oscillations (they grow like $a^{(3 \gamma-5) / 4}$ ) and, for large $a$, they grow like in a cold gas $\left(\delta_{+} \sim a\right)$. Therefore, considering the small $a$ regime, the perturbations grow for $\gamma<4 / 3$, decay for $4 / 3<\gamma<5 / 3$, and grow for $\gamma>5 / 3$. Considering the large $a$ regime, the perturbations decay for $\gamma<4 / 3$ and grow for $\gamma>4 / 3$. For $\gamma<4 / 3$, the perturbations start to grow but finally decay. In a sense, the system is asymptotically stable. However, the growth of perturbations in the initial stage can trigger nonlinear effects that may induce instabilities.

For $\gamma=4 / 3(n=3)$, the density contrast behaves like (see Appendix A):

$\delta_{ \pm}(a) \propto a^{-\frac{1}{4} \pm \sqrt{\frac{25}{16}-\frac{3 k^{2}}{2 k_{\mathrm{J}}^{2}}}}$.

This polytropic index corresponds to a gas of photons at temperature $T$ or to a gas of relativistic fermions at zero temperature. In that case, the Jeans length is independent of time: $k_{\mathrm{J}}=\kappa_{\mathrm{J}}$. For $k>(25 / 24)^{1 / 2} k_{\mathrm{J}}$ the density contrast behaves like

$\delta_{ \pm}(a) \propto \frac{1}{a^{1 / 4}} \cos \left(\sqrt{\frac{3 k^{2}}{2 \kappa_{\mathrm{J}}^{2}}-\frac{25}{16}} \ln a+\phi\right)$.

\footnotetext{
9 In this section, we allow $p$ to be interpreted as ordinary pressure; i.e., we consider not only a gas of BECs, but also a more conventional gas with kinetic pressure.
}

It diverges for $a \rightarrow 0$, implying that we must start the stability analysis at $a_{i}>0$. For $a \geq a_{i}$, the perturbations decay like $a^{-1 / 4}$ while making oscillations. For $k_{\mathrm{J}}<k<(25 / 24)^{1 / 2} k_{\mathrm{J}}$, the perturbations decay algebraically without oscillating. For $k<k_{\mathrm{J}}$, the perturbations grow algebraically (more precisely $\delta_{+}$grows and $\delta_{-}$decays). For $k \rightarrow 0$, the perturbations behave like in a cold gas $\left(\delta_{+}\right.$grows like $a$ and $\delta_{-}$decays like $\left.a^{-2 / 3}\right)$. This situation is relatively close to the classical Jeans stability analysis, but (i) the growth of perturbation is algebraic instead of exponential; (ii) the oscillatory solutions decay with time; (iii) there is a short interval $k_{\mathrm{J}}<k<(25 / 24)^{1 / 2} k_{\mathrm{J}}$ that has no counterpart in the static Jeans analysis.

We now consider particular equations of state with a physical meaning. The index $\gamma=1$ corresponds to an isothermal universe. The density contrast is given by

$\delta_{ \pm}(a) \propto \frac{1}{a^{1 / 4}} J_{ \pm \frac{5}{2}}\left(\sqrt{6} \frac{k}{\kappa_{\mathrm{J}}} a^{1 / 2}\right)$.

For the index $\gamma=5 / 3(n=3 / 2)$, we obtain

$\delta_{ \pm}(a) \propto \frac{1}{a^{1 / 4}} J_{ \pm \frac{5}{2}}\left(\sqrt{6} \frac{k}{\kappa_{\mathrm{J}}} \frac{1}{a^{1 / 2}}\right)$.

This polytropic index corresponds to a gas of nonrelativistic fermions at zero temperature. For the polytropic index $\gamma=2$ $(n=1)$, we have

$\delta_{ \pm}(a) \propto \frac{1}{a^{1 / 4}} J_{ \pm \frac{5}{4}}\left(\sqrt{\frac{3}{2}} \frac{k}{\kappa_{\mathrm{J}}} \frac{1}{a}\right)$.

This polytropic index corresponds to a BEC with quartic selfinteraction in the TF approximation (see Sect. 2). In that case, $\kappa_{\mathrm{J}}=\left(G m^{3} / a_{\mathrm{s}} \hbar^{2}\right)^{1 / 2}$. The previous expression assumes that $a_{\mathrm{s}}>$ 0 . In the case where $a_{\mathrm{s}}<0$, we obtain

$\delta_{ \pm}(a) \propto \frac{1}{a^{1 / 4}} I_{ \pm \frac{5}{4}}\left(\sqrt{\frac{3}{2}} \frac{k}{\kappa_{\mathrm{J}}} \frac{1}{a}\right)$,

with $\kappa_{\mathrm{J}}=\left(G m^{3} /\left|a_{\mathrm{s}}\right| \hbar^{2}\right)^{1 / 2}$. For large $a$, the perturbations behave like in a cold classical gas. For small $a$, we find that

$\delta_{ \pm}(a) \propto a^{1 / 4} \mathrm{e}^{\sqrt{\frac{3}{2} \frac{k}{k_{J}} \frac{1}{a}}} \rightarrow+\infty$.

We can combine the solutions $\delta_{+}(a)$ and $\delta_{-}(a)$ to avoid the divergence at the origin. In particular, the perturbation $\delta(a) \propto$ $\delta_{+}(a)-\delta_{-}(a)$ starts from zero at $a=0$ with a horizontal tangent and rapidly increases. By contrast, in a cold classical gas, a perturbation $\delta(a) \simeq \delta^{\prime}(a) \simeq 0$ at $a=0$ takes a long time to grow. This demonstrates that the formation of structures is faster in BEC dark matter with an attractive self-interaction than in a cold classical universe.

Some curves displaying the evolution of the density contrast $\delta(a)$ in the different cases listed above are represented in Figs. 16 for illustration. The last figure shows the effect of negative scattering on the growth of perturbations.

\subsection{The noninteracting BEC case}

In the noninteracting case $a_{\mathrm{s}}=c_{\mathrm{s}}=0$, corresponding to a BEC universe without self-interaction, Eq. (58) reduces to

$\frac{\mathrm{d}^{2} f}{\mathrm{~d} a^{2}}-\frac{1}{2 a} \frac{\mathrm{d} f}{\mathrm{~d} a}+\frac{1}{a^{2}}\left(\frac{3 k^{4}}{2 \kappa_{Q}^{4} a}-1\right) f=0$. 
P. H. Chavanis: Growth of perturbations in an expanding universe with Bose-Einstein condensate dark matter

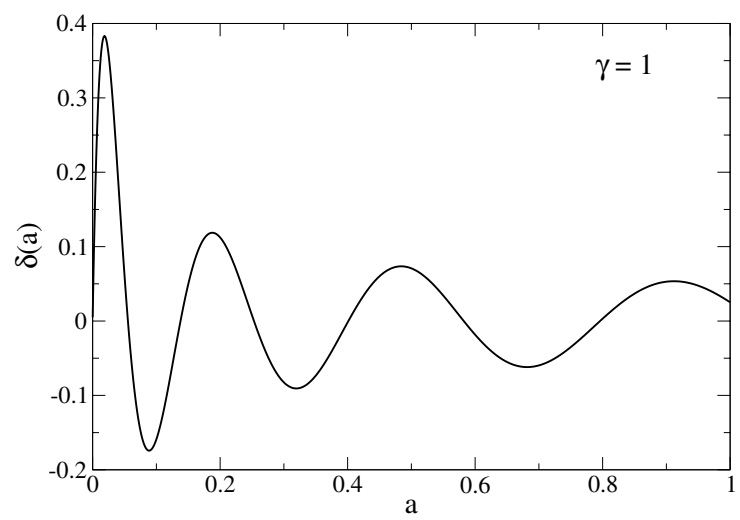

Fig. 1. Evolution of the perturbation $\delta_{+}(a)$ in an isothermal universe $(\gamma=1)$. This corresponds to the case $\gamma<4 / 3$. We have taken $k / k_{\mathrm{J}}=10$.

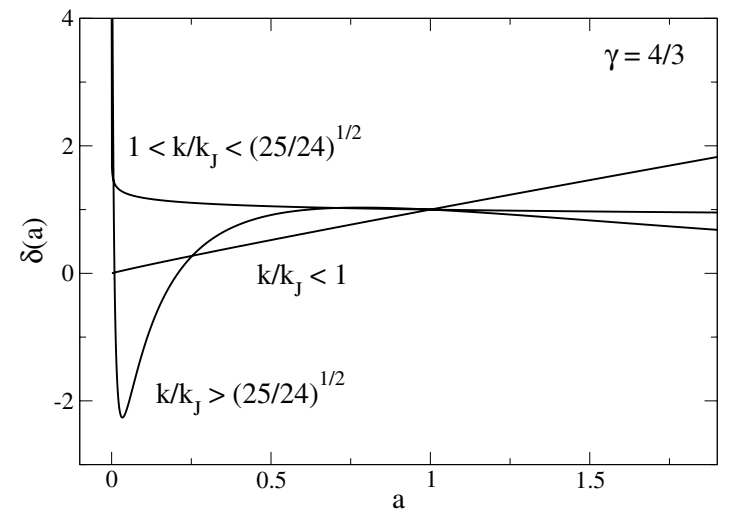

Fig. 2. Evolution of the perturbation $\delta_{+}(a)$ in a photonic or in a relativistic fermionic universe $(\gamma=4 / 3)$.

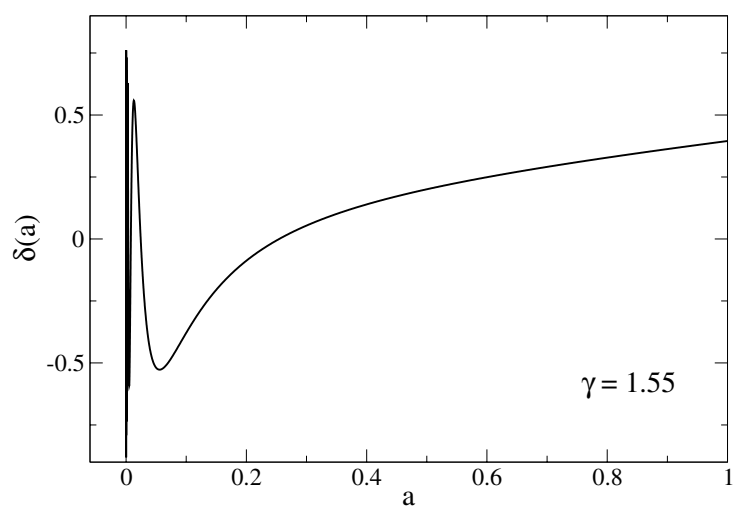

Fig. 3. Evolution of the perturbation $\delta_{+}(a)$ in a polytropic universe with $\gamma=1.55$. This corresponds to the case $4 / 3<\gamma<5 / 3$. We have taken $k / \kappa_{\mathrm{J}}=0.637$.

Comparing this equation with Eq. (59), we see that the dependence on $a$ is the same as for a polytrope $\gamma=5 / 3$. Therefore, $f(a)$ is given by Eq. (A.7) with $\lambda=3 k^{4} / 2 \kappa_{Q}^{4}$ and $\alpha=-1 / 2$ yielding

$\delta_{ \pm}(a) \propto \frac{1}{a^{1 / 4}} J_{ \pm \frac{5}{2}}\left(\sqrt{6} \frac{k^{2}}{\kappa_{Q}^{2}} \frac{1}{a^{1 / 2}}\right)$.

For small $a$, the perturbations oscillate, and, for large $a$, they behave like in a cold gas $\left(\delta_{+}\right.$grows like $\left.a\right)$. In a sense, a cold bosonic universe behaves similarly to a cold nonrelativistic fermionic universe (compare Eqs. (69) and (64)). However, the

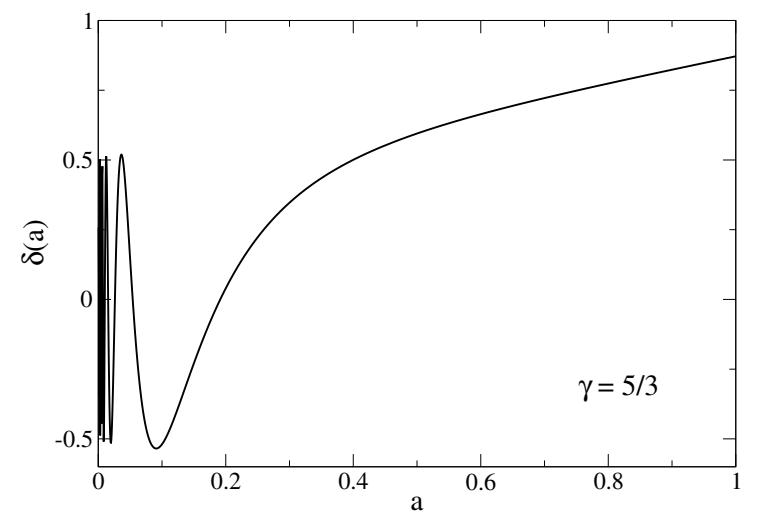

Fig. 4. Evolution of the perturbation $\delta_{+}(a)$ in a nonrelativistic fermionic universe or in a BEC universe without self-interaction $(\gamma=5 / 3)$. We have taken $k / \kappa_{\mathrm{J}}=0.707$.

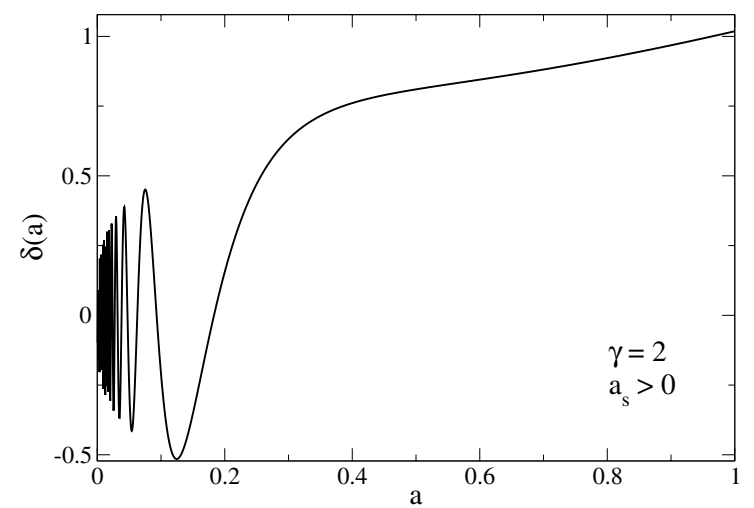

Fig. 5. Evolution of the perturbation $\delta_{+}(a)$ in a BEC universe with repulsive quartic self-interaction in the TF limit $\left(\gamma=2, a_{\mathrm{s}}>0\right)$. This corresponds to the case $\gamma>5 / 3$. We have taken $k / \kappa_{\mathrm{J}}=0.5$.

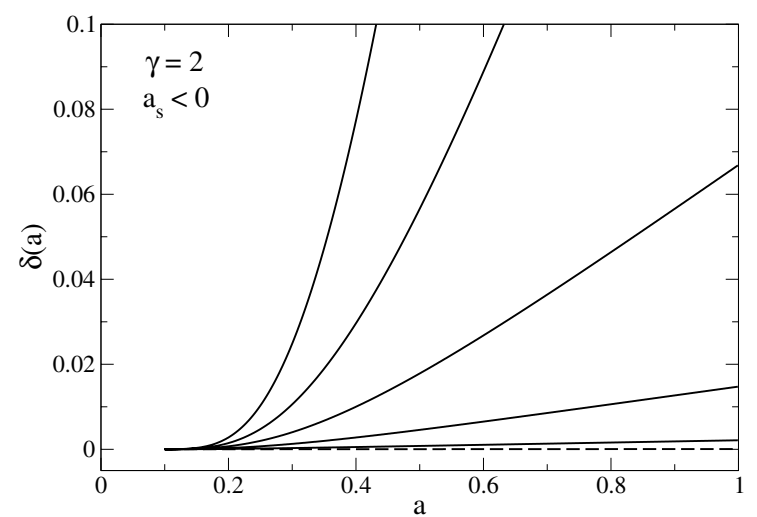

Fig. 6. Evolution of the perturbation $\delta(a)$ in a BEC universe with attractive quartic self-interaction in the TF limit $\left(\gamma=2, a_{\mathrm{s}}<0\right)$. We have numerically solved Eq. (57) with $\kappa_{Q} \rightarrow+\infty$ and $\kappa_{\mathrm{J}} \rightarrow i \kappa_{\mathrm{J}}$, with the initial condition $a_{i}=0.1, \delta\left(a_{i}\right)=10^{-5}$ and $\delta^{\prime}\left(a_{i}\right)=0$. From bottom to top: $k / \kappa_{\mathrm{J}}=0.2,0.4,0.6,0.8,1$. The lowest curve corresponds to a cold classical gas.

dependence on $k$ is different ( $k^{2}$ instead of $k$ ), and the expression of the Jeans length is also different (compare Eqs. (46) and (48)).

\subsection{The nonrelativistic fermionic case $\gamma=5 / 3$}

A nonrelativistic gas of fermions at $T=0$ is usually described by a polytropic equation of state $p=K \rho^{\gamma}$ with index $\gamma=5 / 3$ 
$(n=3 / 2)$ and polytropic constant $K=(1 / 5)(3 / 8 \pi)^{2 / 3} h^{2} / m^{8 / 3}$ (Chandrasekhar 1939). This equation of state arises from the Pauli exclusion principle. Such a semi-classical description is valid in the TF approximation, which is exact when $N \rightarrow+\infty$. In some cases, it can be relevant to go beyond the TF approximation and take the quantum pressure arising from the Heisenberg principle into account. This can regularize the dynamics on small scales, as shown by Bilic et al. (2001) in their study of the formation of fermion stars by gravitational collapse. In our problem, this general situation is described by Eq. (57) with $\gamma=5 / 3$. This yields

$\frac{\mathrm{d}^{2} \delta}{\mathrm{d} a^{2}}+\frac{3}{2 a} \frac{\mathrm{d} \delta}{\mathrm{d} a}+\frac{3}{2 a^{2}}\left(\frac{k^{4}}{\kappa_{Q}^{4} a}+\frac{k^{2}}{\kappa_{\mathrm{J}}^{2} a}-1\right) \delta=0$.

The equation for $f(a)$ is

$\frac{\mathrm{d}^{2} f}{\mathrm{~d} a^{2}}-\frac{1}{2 a} \frac{\mathrm{d} f}{\mathrm{~d} a}+\frac{1}{a^{2}}\left(\frac{3 k^{4}}{2 \kappa_{Q}^{4} a}+\frac{3 k^{2}}{2 \kappa_{\mathrm{J}}^{2} a}-1\right) f=0$.

As noted in Sect. 7.3, the two terms have the same dependence on $a$. Therefore, $f(a)$ is given by Eq. (A.7) with

$\lambda=\frac{3 k^{4}}{2 \kappa_{Q}^{4}}+\frac{3 k^{2}}{2 \kappa_{\mathrm{J}}^{2}}, \quad \alpha=-\frac{1}{2}$.

We obtain

$\delta_{ \pm}(a) \propto \frac{1}{a^{1 / 4}} J_{ \pm \frac{5}{2}}\left(\sqrt{\frac{6 k^{4}}{\kappa_{Q}^{4}}+\frac{6 k^{2}}{\kappa_{\mathrm{J}}^{2}}} \frac{1}{a^{1 / 2}}\right)$.

For small $a$, the perturbations oscillate and, for large $a$, they behave like in a cold gas.

\subsection{The relativistic fermionic case $\gamma=4 / 3$}

A relativistic gas of fermions at $T=0$ is usually described by a polytropic equation of state $p=K \rho^{\gamma}$ with index $\gamma=4 / 3$ $(n=3)$ and polytropic constant $K=(1 / 4)(3 / 8 \pi)^{1 / 3} h c / m^{4 / 3}$ (Chandrasekhar 1939). If we go beyond the TF approximation and take the quantum pressure (Heisenberg) into account, the evolution of the density contrast is described by Eq. (57) with $\gamma=4 / 3$. This yields

$\frac{\mathrm{d}^{2} \delta}{\mathrm{d} a^{2}}+\frac{3}{2 a} \frac{\mathrm{d} \delta}{\mathrm{d} a}+\frac{3}{2 a^{2}}\left(\frac{k^{4}}{\kappa_{Q}^{4} a}+\frac{k^{2}}{\kappa_{\mathrm{J}}^{2}}-1\right) \delta=0$.

The equation for $f(a)$ is

$\frac{\mathrm{d}^{2} f}{\mathrm{~d} a^{2}}-\frac{1}{2 a} \frac{\mathrm{d} f}{\mathrm{~d} a}+\frac{1}{a^{2}}\left(\frac{3 k^{4}}{2 \kappa_{Q}^{4} a}+\frac{3 k^{2}}{2 \kappa_{\mathrm{J}}^{2}}-1\right) f=0$.

Its solution is given by Eq. (A.17), and the evolution of the density contrast is

$\delta_{ \pm}(a) \propto \frac{1}{a^{1 / 4}} J_{ \pm \frac{1}{2}} \sqrt{25-24 \frac{k^{2}}{k_{J}^{2}}}\left(\sqrt{6} \frac{k^{2}}{\kappa_{Q}^{2}} \frac{1}{a^{1 / 2}}\right)$.
We note that the classical Jeans scale appears in the index of the Bessel function, while the quantum Jeans scale appears in the argument of the Bessel function.

\subsection{The general case (asymptotics)}

We now treat the general case, taking quantum pressure and polytropic pressure (we assume $c_{\mathrm{s}}^{2}>0$ ) into account. Since we cannot solve Eq. (57) analytically, we just give asymptotic results.

We first consider the small $a$ regime. (i) If $\gamma<5 / 3$, the polytropic pressure is negligible in front of the quantum pressure and the perturbations oscillate (see Sect. 7.3). (ii) If $\gamma=5 / 3$, the polytropic pressure and the quantum pressure are of the same order of magnitude, and the perturbations oscillate (see Sect. 7.4). (iii) If $\gamma>5 / 3$, the quantum pressure is negligible in front of the polytropic pressure and the perturbations undergo growing oscillations $\delta \propto a^{(3 \gamma-5) / 4}$ (see Sect. 7.2). Therefore, the perturbations oscillate for $\gamma \leq 5 / 3$ and grow for $\gamma>5 / 3$. The quantum pressure plays a stabilizing role for $\gamma \leq 4 / 3$ (see Sect. 7.2).

We now consider the large $a$ regime. (i) If $\gamma<4 / 3$, the quantum pressure is negligible in front of the polytropic pressure, and the perturbations undergo damped oscillations and decay like $1 / a^{(5-3 \gamma) / 4}$ (see Sect. 7.2). (ii) If $\gamma=4 / 3$, the quantum pressure is negligible in front of the polytropic pressure, and we are led back to the critical case of Sect. 7.2: for $k>(25 / 24)^{1 / 2} k_{\mathrm{J}}$ the perturbations decay like $a^{-1 / 4}$ while making oscillations, for $k_{\mathrm{J}}<k<(25 / 24)^{1 / 2} k_{\mathrm{J}}$ the perturbations decay algebraically without oscillating and for $k<k_{\mathrm{J}}$ the perturbations grow algebraically. (iii) For $\gamma>4 / 3$, the perturbations behave like in a cold gas and grow like $\delta_{+} \sim a$. Therefore, the perturbations decay for $\gamma<4 / 3$ and grow for $\gamma>4 / 3$. In conclusion, the system is asymptotically stable for $\gamma<4 / 3$ and asymptotically unstable for $\gamma>4 / 3$. For the critical index $\gamma=4 / 3$, the system is stable for $k>k_{\mathrm{J}}$ and unstable for $k<k_{\mathrm{J}}$.

\subsection{Formation of BEC dark matter halos}

The results of this stability analysis lead to the following scenario. For a gas with polytropic index $\gamma>4 / 3$, including the BEC dark matter model corresponding to $\gamma=2$, an expanding homogeneous distribution of matter is gravitationally unstable to small perturbations and a process of fragmentation follows. In the linear regime, the perturbations grow and overdense regions appear. At first, these regions experience a cold collapse and intensify. Then, when pressure effects become important at high densities and small scales, localized clusters in virial equilibrium (DM halos) form. These clusters, which correspond to complete polytropic spheres, are stable according to the general stability criterion $\gamma>4 / 3$ recalled at the end of Sect. 6.1. Therefore, this scenario explains the formation of BEC dark matter halos by Jeans instability in an expanding spatially homogeneous gas, and their stabilization by pressure effects when they become dense enough. The weakly nonlinear gravitational clustering (intermediate regime) could be described by resorting to the Zeldovich approximation, incorporating the properties of the BEC (see Chavanis 2011b).

Remark: an expanding homogeneous gas is stable if $\gamma<4 / 3$ and unstable if $\gamma>4 / 3$, leading to fragmentation and structure formation. Inversely, an isolated dense cluster (dark matter halo or star) is stable if $\gamma>4 / 3$ and unstable if $\gamma<4 / 3$. Finally, a collapsing homogeneous gas is stable if $\gamma>4 / 3$ and unstable if $\gamma<4 / 3$, leading to fragmentation (Chavanis 2011c). 


\section{Bose-Einstein condensate universe}

\subsection{Newtonian cosmology with pressure}

We now take pressure effects (coming from special relativity) into account in the evolution of the cosmic fluid. We base our study on the set of hydrodynamic equations

$$
\begin{aligned}
& \frac{\partial \rho}{\partial t}+\nabla \cdot(\rho \boldsymbol{u})+\frac{p}{c^{2}} \nabla \cdot \boldsymbol{u}=0, \\
& \frac{\partial \boldsymbol{u}}{\partial t}+(\boldsymbol{u} \cdot \nabla) \boldsymbol{u}=-\frac{\nabla p}{\rho+\frac{p}{c^{2}}}-\nabla \Phi, \\
& \Delta \Phi=4 \pi G\left(\rho+\frac{3 p}{c^{2}}\right)-\Lambda,
\end{aligned}
$$

where $c$ is the velocity of light. For $c \rightarrow+\infty$, we recover the classical Euler-Poisson system (Binney \& Tremaine 1987). These equations were introduced by McCrea (1951). His initial continuity equation, which contained an incorrect pressure gradient term, was corrected by Lima et al. (1997), and we considered this correction in writing Eq. (77). These equations lead to the correct relativistic equations for the cosmic evolution. Indeed, assuming a homogeneous and isotropic solution of the form $\rho(\boldsymbol{r}, t)=\rho_{\mathrm{b}}(t)$, $p(\boldsymbol{r}, t)=p_{\mathrm{b}}(t)$, and $\boldsymbol{u}(\boldsymbol{r}, t)=(\dot{a} / a) \boldsymbol{r}$, Eqs. (77)-(79) reduce to

$$
\begin{aligned}
& \frac{\mathrm{d} \rho_{\mathrm{b}}}{\mathrm{d} t}+3 \frac{\dot{a}}{a}\left(\rho_{\mathrm{b}}+\frac{p_{\mathrm{b}}}{c^{2}}\right)=0, \\
& \frac{\ddot{a}}{a}=-\frac{4 \pi G}{3}\left(\rho_{\mathrm{b}}+\frac{3 p_{\mathrm{b}}}{c^{2}}\right)+\frac{\Lambda}{3}, \\
& \left(\frac{\dot{a}}{a}\right)^{2}=\frac{8 \pi G}{3} \rho_{\mathrm{b}}-\frac{\kappa}{a^{2}}+\frac{\Lambda}{3} .
\end{aligned}
$$

These are precisely the Friedmann equations that can be derived from the theory of general relativity (Weinberg 1972). The perturbation theory based on Eqs. (77)-(79) has been discussed by Reis (2003), who show that, depending on the equation of state, the results may agree or disagree with the general relativistic approach. We leave this problem open since it is not our purpose here to investigate the validity of these equations in detail. Furthermore, our main results concern the evolution of the cosmic fluid governed by the Friedmann Eqs. (80)-(82). Since the Friedmann equations can be derived from the theory of general relativity, the validity of these equations is not questioned. Equations different from Eqs. (77)-(79) have been considered by Pace et al. (2010) and Harko (2011a). Their equations involve an additional term $\dot{\boldsymbol{p}} \boldsymbol{u} / c^{2}$ in the Euler Eq. (78). However, this term apparently leads to equations for the cosmic evolution that are different from the Friedmann equations, so this term is not considered here.

\subsection{The cosmic evolution of a BEC universe}

We assume that dark matter is a BEC with quartic selfinteraction ${ }^{10}$ described by the barotropic equation of state (8). For convenience, we write this equation in the form

$p=k c^{2} \rho^{2}$,

where $k=2 \pi a_{\mathrm{s}} \hbar^{2} / m^{3} c^{2}$ is a constant that can be positive $\left(a_{\mathrm{s}}>\right.$ $0)$ or negative $\left(a_{\mathrm{s}}<0\right)$. In a first step, we concentrate on the dark matter component and neglect radiation, baryonic matter, and dark energy (in particular we take $\Lambda=0$ ). These additional

\footnotetext{
${ }^{10}$ In this part of the paper, we neglect the quantum pressure.
}

terms are considered later. For the equation of state (83), the Friedmann Eq. (80) becomes

$\frac{\mathrm{d} \rho_{\mathrm{b}}}{\mathrm{d} t}+3 \frac{\dot{a}}{a} \rho_{\mathrm{b}}\left(1+k \rho_{\mathrm{b}}\right)=0$.

This equation can be integrated into

$\rho_{\mathrm{b}}=\frac{A}{a^{3}-k A}$,

where $A$ is a constant. If we make the physical requirement that Eq. (85) has solutions for large $a$, we must impose $A>0$. In that case, $\rho_{\mathrm{b}} \sim A / a^{3}$ for $a \rightarrow+\infty$ which returns Eq. (18). When $k>$ 0 , which is the case previously considered by Harko (2011a), the density exists only for $a>a_{*}=(k A)^{1 / 3}$. For $a \rightarrow a_{*}, \rho_{\mathrm{b}} \rightarrow+\infty$. When $k<0$, the density is defined for all $a$ and has a finite value $\rho_{\mathrm{b}}=1 /|k|$ when $a \rightarrow 0$.

Combining Eqs. (81) and (83), we obtain

$\frac{\ddot{a}}{a}=-\frac{4 \pi G \rho_{\mathrm{b}}}{3}\left(1+3 k \rho_{\mathrm{b}}\right)$.

When $k>0$, the universe is always decelerating $(\ddot{a}<0)$. When $k<0$, the universe is accelerating ( $\ddot{a}>0$ ) for $\rho_{\mathrm{b}}>\rho_{\mathrm{c}} \equiv 1 / 3|k|$ and decelerating $(\ddot{a}<0)$ for $\rho_{\mathrm{b}}<\rho_{\mathrm{c}}$. Using Eq. (85), this can be expressed in terms of the radius ${ }^{11}$ : the universe is accelerating for $a<a_{\mathrm{c}} \equiv(2 A|k|)^{1 / 3}$ and decelerating for $a>a_{\mathrm{c}}$.

To determine the temporal evolution of $a(t)$, we assume $\kappa=$ 0 (flat space) as in the Einstein-de Sitter universe. Combining Eqs. (82) and (85), we get

$\dot{a}=\left(\frac{8 \pi G A}{3}\right)^{1 / 2} \frac{a}{\sqrt{a^{3}-k A}}$.

We note that, for $a \rightarrow+\infty$, this equation reduces to Eq. (22) with Eq. (18) so we recover the Einstein-de Sitter solution (23). It is convenient to define $a_{*}=(|k| A)^{1 / 3}$ and introduce $R=a / a_{*}$. In that case, the density is given by

$\rho_{\mathrm{b}}=\frac{1}{|k|} \frac{1}{R^{3} \mp 1}$

and Eq. (87) can be rewritten as

$\dot{R}=\frac{K R}{\sqrt{R^{3} \mp 1}}$,

where the upper sign - corresponds to $k>0$ and the lower sign + corresponds to $k<0$. We have defined the constant

$K=\left(\frac{8 \pi G}{3|k|}\right)^{1 / 2}=\left(\frac{4 G m^{3} c^{2}}{3 \mid a_{\mathrm{s}} \hbar^{2}}\right)^{1 / 2}$,

which depends on the mass $m$ of the bosons and on their scattering length $a_{\mathrm{s}}$. Introducing the dimensionless parameter $\lambda / 8 \pi=$ $a_{\mathrm{s}} / \lambda_{\mathrm{c}}=a_{\mathrm{s}} m c / \hbar$ (Chavanis 2011a) measuring the strength of the short-range interactions $\left(\lambda_{\mathrm{c}}\right.$ is the Compton wavelength of the bosons), we can rewrite Eq. (90) in the form

$K=\frac{2}{\sqrt{3}}\left(\frac{m}{M_{\mathrm{P}}}\right)^{2} \sqrt{\frac{8 \pi}{|\lambda|}} t_{\mathrm{P}}^{-1}$,

${ }^{11}$ We sometimes call the scale factor $a$ the "radius", keeping in mind the naive picture of a universe behaving like a homogeneous sphere of radius $a$. This is, of course, an abuse of language in the context of the theory of general relativity. 


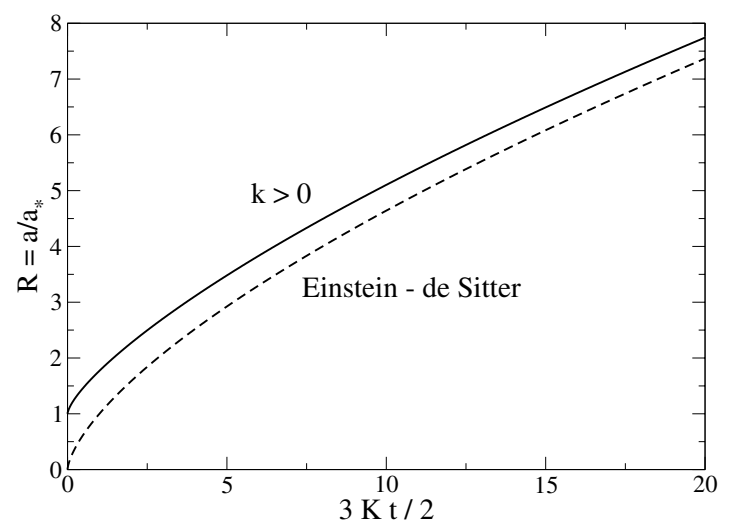

Fig. 7. Evolution of the scale factor in a BEC universe with $k>0$. The dashed line corresponds to the Einstein-de Sitter universe $(k=0)$ that is reached asymptotically.

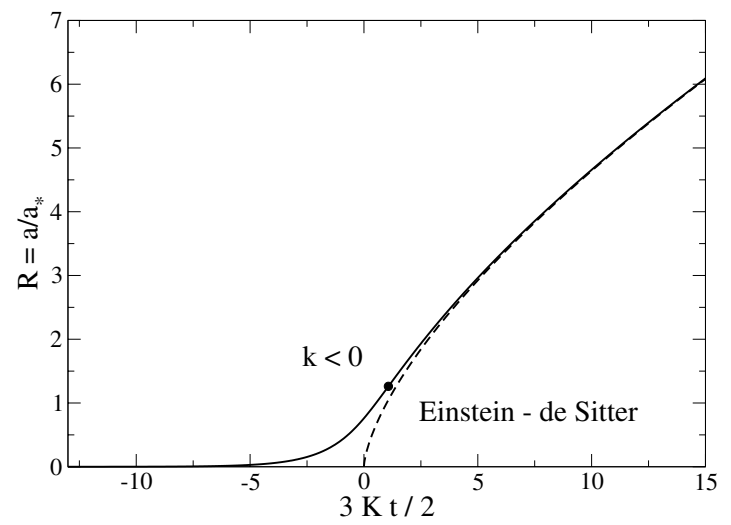

Fig. 8. Evolution of the scale factor in a BEC universe with $k<0$. The dashed line corresponds to the Einstein-de Sitter universe $(k=0)$. The bullet (locating the inflexion point) corresponds to the time $t_{\mathrm{c}}$ at which the universe starts to decelerate.

where $M_{\mathrm{P}}=(\hbar c / G)^{1 / 2}$ is the Planck mass, $l_{\mathrm{P}}=\left(\hbar G / c^{3}\right)^{1 / 2}$ the Planck length, and $t_{\mathrm{P}}=l_{\mathrm{P}} / c$ the Planck time.

For $k>0$, which is the case previously considered by Harko (2011a), the solution of Eq. (89) is

$$
\sqrt{R^{3}-1}-\arctan \sqrt{R^{3}-1}=\frac{3}{2} K t,
$$

where the constant of integration has been set equal to zero. In this model, the universe starts at a finite time $t=0$ (see Sect. 8.5 for a revision of this statement) with a finite radius $R(0)=1$ and an infinite density $\rho_{\mathrm{b}}(0)=\infty$. For $t \rightarrow 0, R \simeq 1+(3 / 4)^{1 / 3}(K t)^{2 / 3}$. The universe is expanding, always decelerating, and asymptotically approaching the Einstein-de Sitter universe $R \sim(3 K t / 2)^{2 / 3}$ for $t \rightarrow+\infty$ (see Fig. 7).

For $k<0$, the solution of Eq. (89) is

$$
\sqrt{R^{3}+1}-\ln \left(\frac{1+\sqrt{R^{3}+1}}{R^{3 / 2}}\right)=\frac{3}{2} K t,
$$

where the constant of integration has been set equal to zero. In this model, the universe starts from $t \rightarrow-\infty$ with a vanishing radius $R(-\infty)=0$ and a finite density $\rho_{\mathrm{b}}(-\infty)=1 /|k|$. For $R<R_{\mathrm{c}} \equiv 2^{1 / 3} \simeq 1.26$, the universe is accelerating and decelerating for $R>R_{\mathrm{c}} \equiv 2^{1 / 3}$. For $R \rightarrow+\infty$, we recover the classical Einstein-de Sitter universe $R \sim(3 K t / 2)^{2 / 3}$ (see Fig. 8). We note that a BEC universe with $k<0$ converges more rapidly towards the EdS solution than a BEC universe with $k>0$. The change of regime between the phase of acceleration and the phase of deceleration manifests itself by an inflexion point $(\ddot{R}=0)$. The time $t_{\mathrm{c}}$ at which the universe starts decelerating is given by

$\frac{3}{2} K t_{\mathrm{c}}=\sqrt{3}-\ln \left(\frac{1+\sqrt{3}}{\sqrt{2}}\right) \simeq 1.074$.

At $t=0, R(0) \simeq 0.7601$, and $\rho_{\mathrm{b}}(0)=0.6948 /|k|$. For $t \rightarrow-\infty$, $R \rightarrow 0$ and the asymptotic expansion of Eq. (93) yields

$R(t) \propto \mathrm{e}^{K t}$.

The universe expands exponentially rapidly for $t \ll t_{\mathrm{c}}$, decelerates after $t_{\mathrm{c}}$, and coincides with the Einstein-de Sitter universe for $t \gg t_{\mathrm{c}}$. In the accelerating phase, the rate of the expansion is given by Eq. (90).

Remark: in our mathematical description of the evolution of the cosmic fluid, we have extrapolated the solutions (92) and (93) far away in the past. In the model with $k>0$, the universe emerges at a primordial time $t=0$ from a "big-bang" singularity. Its initial density is infinite, and its radius is strictly positive. In the model with $k<0$, the universe has always existed in the past (up to $t \rightarrow-\infty$ ), and there is no big-bang singularity at $t=0$. This is because the pressure is negative, allowing for the presence of an inflexion point that changes the concavity of the curve $a(t)$ (the proof of the necessity of a big-bang singularity given by Weinberg (1972) assumes that $p \geq 0$ ). Of course, this extrapolation to $t \rightarrow-\infty$ is not physically justified since we have ignored important effects like the radiation that dominates in the early universe. We show in Sect. 8.4 that the contribution of the radiation strongly alters the results at early times in the case $k<0$. Therefore, our description of the solution (93) must only be considered on a formal mathematical basis. Still, it shows that one can construct self-consistent cosmological models, that are solution to the Einstein equations, without primordial (big-bang) singularity when the pressure is negative. They correspond to eternal universes in the future and past, without an origin. They could be called "aioniotic" universes.

\subsection{Other representations}

The previous representation shows that there is a single universal curve $a(t)$ in each case $a_{\mathrm{s}}>0, a_{\mathrm{s}}=0(\mathrm{EdS})$, and $a_{\mathrm{s}}<0$, provided that the units of time and length are appropriately chosen. However, these units depend on $a_{\mathrm{s}}$. Alternatively, it may be useful to choose units of time and length that are independent of $a_{\mathrm{s}}$ and plot the curve $a(t)$ for different values of $a_{\mathrm{s}}$. This is the representation that has been chosen by Harko (2011a) for the case $k>0$ and that we discuss and generalize in this section.

In Eq. (85), the constant $A$ can be determined by the presentday density $\rho_{\mathrm{b}}=\rho_{0}$ and the corresponding scale factor $a=a_{0}$. Writing $\rho_{0}=A /\left(a_{0}^{3}-k A\right)$, we obtain

$A=\frac{\rho_{0} a_{0}^{3}}{1+k \rho_{0}}$.

We note that $\rho_{0}<1 /|k|$ when $k<0$, as we have already indicated. The relation (85) between the density and the scale factor can be rewritten

$\rho_{\mathrm{b}}=\frac{q}{k} \frac{1}{\left(\frac{a}{a_{0}}\right)^{3}-q}$,

where we have introduced the dimensionless parameter

$q=\frac{k \rho_{0}}{1+k \rho_{0}}$. 
P. H. Chavanis: Growth of perturbations in an expanding universe with Bose-Einstein condensate dark matter

We note that $q$ is positive for $k>0$ and negative for $k<0$. Furthermore, $q=0$ for $k=0, q \rightarrow 1$ for $k \rightarrow+\infty$ and $q \rightarrow-\infty$ for $k \rightarrow-1 / \rho_{0}$. We use $q$ as a control parameter. Therefore, $k$ must be expressed in terms of $q$ according to

$k \rho_{0}=\frac{q}{1-q}$.

The relation (97) between the density and the scale factor becomes

$\rho_{\mathrm{b}}=\rho_{0} \frac{1-q}{\left(\frac{a}{a_{0}}\right)^{3}-q}$,

Using these results, the Friedmann Eq. (82) giving the temporal evolution of the scale factor takes the form (for $\kappa=\Lambda=0$ ):

$\dot{a}=\sqrt{\frac{8 \pi G \rho_{0}}{3}}(1-q)^{1 / 2} \frac{a}{\sqrt{\left(\frac{a}{a_{0}}\right)^{3}-q}}$.

Quite generally, from Eq. (82), we can define the present-day critical density by $\left(\rho_{\mathrm{c}}\right)_{0}=3 H_{0}^{2} / 8 \pi G$ where $H_{0}$ is the present-day Hubble constant. If $\kappa=0$, which seems to be the case for our universe, $\left(\rho_{\mathrm{c}}\right)_{0}$ represents the total density including baryonic matter, radiation, dark matter and dark energy. It is customary to introduce the present-day density parameter $\Omega_{0}=\rho_{0} /\left(\rho_{\mathrm{c}}\right)_{0}$. Then, Eq. (101) becomes

$\dot{a}=H_{0} \sqrt{\Omega_{0}}(1-q)^{1 / 2} \frac{a}{\sqrt{\left(\frac{a}{a_{0}}\right)^{3}-q}}$.

We can make the connection with the notations of Sect. 8.2 by setting

$a_{*}=a_{0}|q|^{1 / 3}, \quad K=H_{0} \sqrt{\Omega_{0}}\left(\frac{1-q}{|q|}\right)^{1 / 2}$.

In that case, Eq. (102) takes the form of Eq. (89) and its solutions are given by Eqs. (92) and (93). Returning to the notations of this section, we find for $0 \leq q<1$ that

$$
\begin{array}{r}
\frac{1}{\sqrt{1-q}}\left\{\sqrt{\left(\frac{a}{a_{0}}\right)^{3}-q}-\sqrt{q} \arctan \sqrt{\frac{\left(\frac{a}{a_{0}}\right)^{3}-q}{q}}\right\} \\
=\frac{3}{2} \sqrt{\Omega_{0}} H_{0} t,
\end{array}
$$

which was previously obtained by Harko (2011a). On the other hand, for $q \leq 0$, we obtain

$$
\begin{gathered}
\frac{1}{\sqrt{1-q}}\left\{\sqrt{\left(\frac{a}{a_{0}}\right)^{3}-q}-\sqrt{|q|} \ln \left(\frac{\sqrt{|q|}+\sqrt{\left(\frac{a}{a_{0}}\right)^{3}-q}}{\left(\frac{a}{a_{0}}\right)^{3 / 2}}\right)\right\} \\
=\frac{3}{2} \sqrt{\Omega_{0}} H_{0} t .
\end{gathered}
$$

Finally, for $q=0$, we recover the Einstein-de Sitter model

$\frac{a}{a_{0}}=\left(\frac{3}{2} \sqrt{\Omega_{0}}\right)^{2 / 3}\left(H_{0} t\right)^{2 / 3}$.

The curves giving the scale factor $a(t)$ as a function of time for different values of $q$ are plotted in Figs. 9 and 10. The interest of this representation is that it shows how the BEC models (104) and (105) approach the Einstein-de Sitter model (106) as $q \rightarrow 0$.

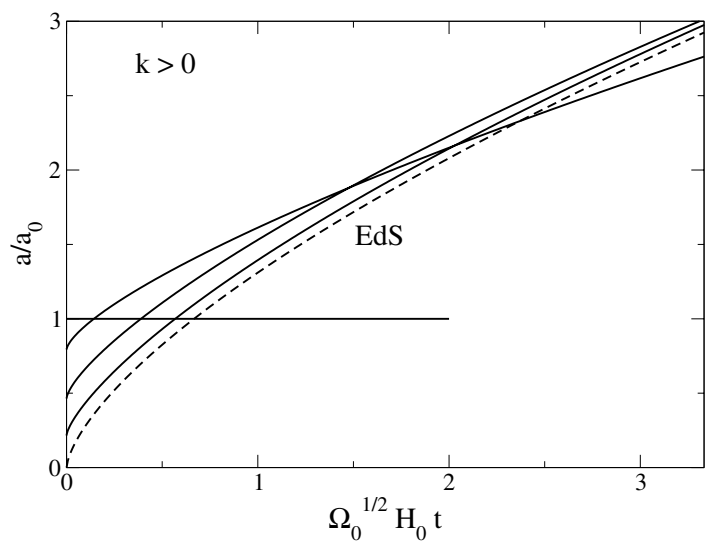

Fig. 9. Scale factor $a(t)$ as a function of time $t$ for a BEC universe with $k \geq 0$. The units of space and time are normalized by present-day quantities that do not depend on $k$. The different curves correspond to $q=0$ (EdS, dashed), $q=0.01, q=0.1$ and $q=0.5$. The straight line corresponds to $a / a_{0}=1$. Its intersection with the curve $a(t)$ defines the age of the universe.

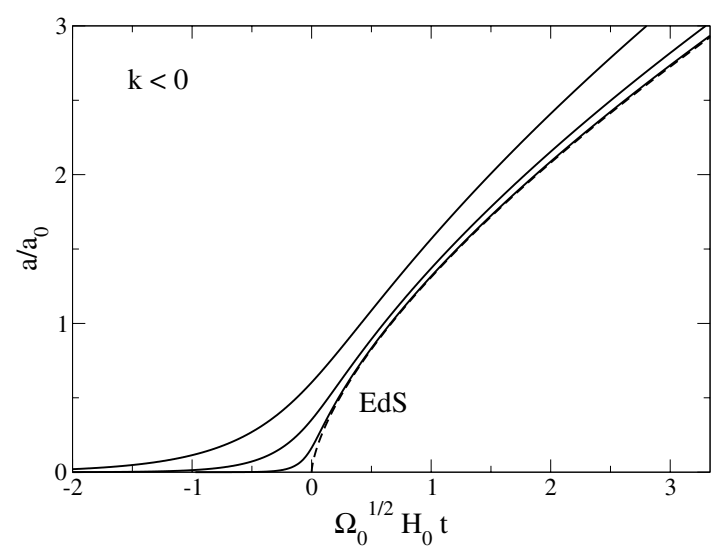

Fig. 10. Scale factor $a(t)$ as a function of time $t$ for a BEC universe with $k \leq 0$. The units of space and time are normalized by present-day quantities that do not depend on $k$. The different curves correspond to $q=0$ (EdS, dashed), $q=-0.01, q=-0.1$, and $q=-0.5$.

On the other hand, for high values of $q$ (in absolute value), there can be substantial differences between a BEC universe and the classical pressureless Einstein-de Sitter universe.

To see that, we consider the asymptotic behaviors of Eqs. (104) and (105). For $t=0$, we find that

$\frac{a}{a_{0}}=R_{ \pm}|q|^{1 / 3}$,

with $R_{+}=1$ for $k>0$ and $R_{-} \simeq 0.7601$ for $k<0$. For $t \rightarrow+\infty$, we get

$\frac{a}{a_{0}} \sim\left(\frac{3}{2} \sqrt{\Omega_{0}}\right)^{2 / 3}(1-q)^{1 / 3}\left(H_{0} t\right)^{2 / 3}$.

For $0<q<1$, the scale factor is asymptotically smaller than in an EdS universe (corresponding to $q=0$ ). Since a BEC universe with $k>0$ initially starts with a radius $a(0)>0$, this implies that the curves $a_{\mathrm{BEC}}(t)$ and $a_{\mathrm{EdS}}(t)$ must cross each other at some point (such a crossing is shown in Fig. 9 for $q=0.5$ ). Of course, the crossing point occurs at later and later times as $q \rightarrow 0$. For $q<0$, the scale factor is always larger than in an EdS universe (see Fig. 10). 


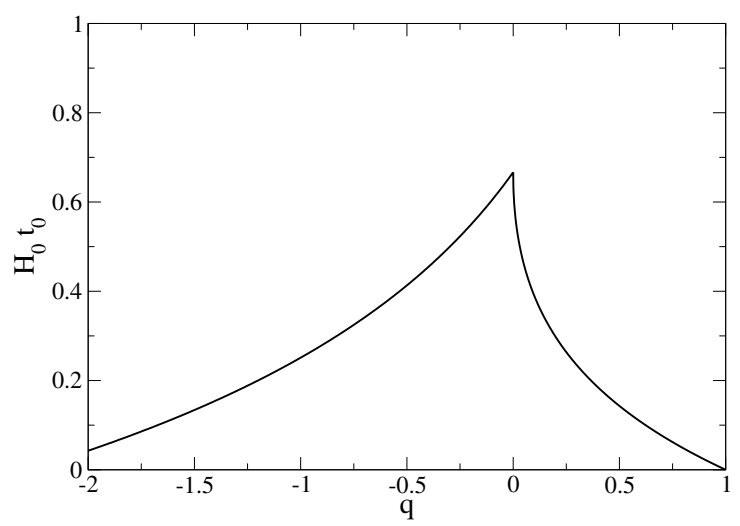

Fig. 11. Age of a BEC universe as a function of $q$. The Einstein-de Sitter model corresponds to $q=0$.

For $q<0$, the inflexion point indicating when the universe starts decelerating is located at

$\frac{a_{\mathrm{c}}}{a_{0}}=2^{1 / 3}|q|^{1 / 3}$

$\frac{3}{2} \sqrt{\Omega_{0}} H_{0} t_{\mathrm{c}}=\left[\sqrt{3}-\ln \left(\frac{1+\sqrt{3}}{\sqrt{2}}\right)\right]\left(\frac{|q|}{1-q}\right)^{1 / 2}$.

For $q \rightarrow-\infty, a_{\mathrm{c}} \rightarrow+\infty$ and $\frac{3}{2} \sqrt{\Omega_{0}} H_{0} t_{\mathrm{c}} \rightarrow 1$ 1.074. On the other hand, for $t \rightarrow-\infty$, the scale factor behaves like

$\frac{a}{a_{0}} \propto e^{\sqrt{\frac{1+|q|}{|q|} \Omega_{0}} H_{0} t}$.

We naively assume that all the content of the universe is in the form of BEC dark matter and take $\Omega_{0}=1$. The age of a BEC universe corresponds to the time $t_{0}$ at which $a / a_{0}=1$. For $0 \leq$ $q<1$, we obtain

$H_{0} t_{0}=\frac{2}{3}\left\{1-\sqrt{\frac{q}{1-q}} \arctan \sqrt{\frac{1-q}{q}}\right\}$,

and, for $q \leq 0$, we find that ${ }^{12}$

$H_{0} t_{0}=\frac{2}{3}\left\{1-\sqrt{\frac{-q}{1-q}} \ln (\sqrt{-q}+\sqrt{1-q})\right\}$.

The age of the EdS universe $(q=0)$ is

$H_{0} t_{0}=\frac{2}{3}$.

As can be seen in Fig. 11, the age of a BEC universe is always less than the age of the EdS universe $(q=0)$. We see in the following section that when the contribution of radiation, baryons, and dark energy is taken into account, the results change qualitatively.

\subsection{Contribution of radiation, baryons and dark energy}

Several observational results indicate that the universe is flat $(\kappa=0)$ and that its present-day expansion is accelerating $(\ddot{a}>0)$. Furthermore, the estimated age of the universe is

${ }_{12}$ In fact, the age of a BEC universe with $q<0$ is formally infinite since there is no primordial singularity. However, Eq. (113) gives its age from the time $t=0$ corresponding to the origin of the EdS universe.

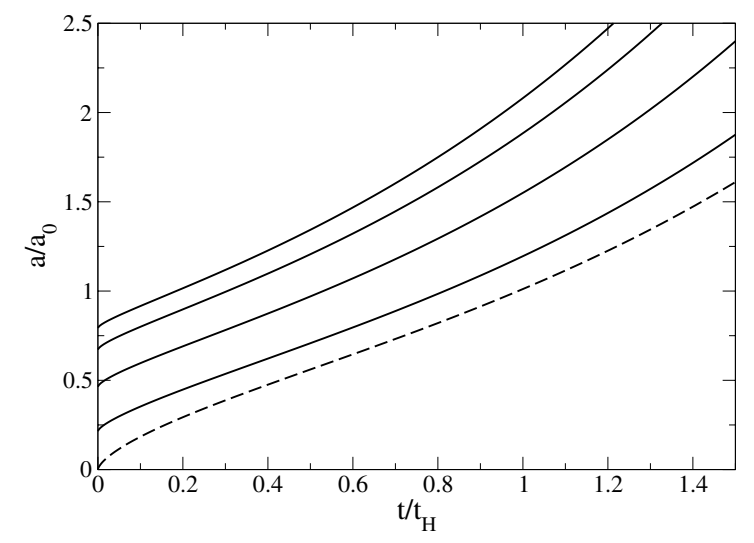

Fig. 12. Scale factor $a(t)$ as a function of time $t$ for a universe filled with baryonic matter, radiation, BEC dark matter with $k \geq 0$, and dark energy. The different curves correspond to $q=0$ (pressureless dark matter, dashed), $q=0.01, q=0.1, q=0.303471$, and $q=0.5$.

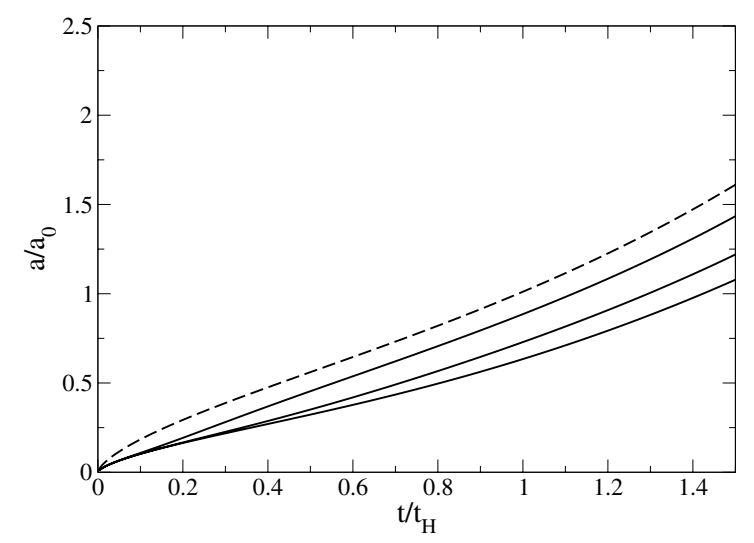

Fig. 13. Scale factor $a(t)$ as a function of time $t$ for a universe filled with baryonic matter, radiation, BEC dark matter with $k \leq 0$, and dark energy. The different curves correspond to $q=0$ (pressureless dark matter, dashed), $q=-0.01, q=-0.1$, and $q=-0.5$.

$t_{0} \sim 13.75 \mathrm{Gyrs}$, which is about $H_{0}^{-1}$. If we assume that the universe is only made of a pressureless fluid, and if we take $\Lambda=0$, we are led to the EdS universe. However, this model leads to a decelerating expansion and predicts a too young age of the universe $t_{0}=2 / 3 H_{0} \sim 9.3 \mathrm{Gyr}$. If we assume that dark matter is a BEC, we again find that the present-day universe is decelerating. Furthermore, its predicted age is even less than the EdS universe (see Sect. 8.3). Therefore, we must invoke some form of dark energy to understand the present-day acceleration of the universe and its age. In the standard $\Lambda$ CDM model, dark energy is due to the cosmological constant. On the other hand, to be complete, we must include the contribution of dark matter, baryonic matter, and radiation. In that case, Eq. (102) is replaced by

$\frac{\dot{a}}{a}=H_{0} \sqrt{\frac{\Omega_{\mathrm{B}, 0}}{\left(a / a_{0}\right)^{3}}+\frac{\Omega_{\mathrm{rad}, 0}}{\left(a / a_{0}\right)^{4}}+\frac{\Omega_{\mathrm{DM}, 0}(1-q)}{\left(a / a_{0}\right)^{3}-q}+\Omega_{\Lambda}}$,

where $\Omega_{\mathrm{B}, 0}, \Omega_{\mathrm{rad}, 0}, \Omega_{\mathrm{DM}, 0}$, and $\Omega_{\Lambda}$ are the present-day values of the density parameters of the baryonic matter, radiation, dark matter, and dark energy. We have assumed that dark matter is in the form of BEC. The case of a pressureless dark matter is recovered for $q=0$. Following Harko (2011a), we adopted the numerical values $\Omega_{\mathrm{B}, 0}=0.0456, \Omega_{\mathrm{rad}, 0}=8.24 \times 10^{-5}, \Omega_{\mathrm{DM}, 0}=$ 0.228 , and $\Omega_{\Lambda}=0.726$ (Hinshaw et al. 2009).

The evolution of the scale factor $a(t)$ is represented in Figs. 12 and 13 for $k>0$ and $k<0$, respectively. In both 
cases, the universe is initially decelerating but finally enters into a phase of acceleration due to the effect of dark energy (cosmological constant). In the case $k>0$, previously treated by Harko (2011a), the scale factor increases more rapidly than in the standard model $(k=0)$, so this universe is younger. Furthermore, the density of dark matter becomes infinite at a finite radius $a_{*}$ so the $\mathrm{BEC}$ energy contribution dominates the radiation and the other cosmological energy terms in the early universe. Accordingly, the universe starts from $a(0)=a_{*}>0$ at $t=0$. In the case $k<0$, the scale factor increases less rapidly than in the standard model $(k=0)$, so this universe is older. Furthermore, the density of dark matter does not diverge when $a \rightarrow 0$, so that radiation dominates in the early universe. Accordingly, the universe starts from $a(0)=0$ with an infinite (radiation) density.

\subsection{Theory of linear perturbations}

The theory of linear perturbations based on the Newtonian equations with pressure (77)-(79) has been developed by Reis (2003) in the general case, and we directly apply his results to the present situation. Harko (2011a) considers other equations and obtains different results. Nevertheless, our main conclusions are the same. For an arbitrary equation of state, the evolution of the density contrast is given by (Reis 2003):

$$
\begin{aligned}
& \ddot{\delta}-H\left[3\left(2 w-c_{\mathrm{eff}}^{2}-c_{\mathrm{s}}^{2}\right)-2\right] \dot{\delta} \\
& +3 H^{2}\left[\frac{1}{H} c_{\mathrm{eff}}^{2}+\left(3 c_{\mathrm{s}}^{2}-6 w-1\right) c_{\mathrm{eff}}^{2}\right. \\
& \left.+\frac{3 w^{2}}{2}-4 w-\frac{1}{2}+3 c_{\mathrm{s}}^{2}\right] \delta-\frac{c_{\mathrm{eff}}^{2} c^{2}}{a^{2}} \Delta \delta=0,
\end{aligned}
$$

where $H=\dot{a} / a$ is the Hubble constant, $w=p_{\mathrm{b}} / \rho_{\mathrm{b}} c^{2}, c_{\mathrm{eff}}^{2}=$ $(\delta p / \delta \rho) / c^{2}$, and $c_{\mathrm{s}}^{2}=p_{\mathrm{b}}^{\prime}\left(\rho_{\mathrm{b}}\right) / c^{2}$. These last quantities represent the velocity of sound normalized by the velocity of light. For a barotropic fluid, $c_{\text {eff }}^{2}=c_{\mathrm{s}}^{2}=p^{\prime}\left(\rho_{\mathrm{b}}\right) / c^{2}$. On the other hand, from the Friedmann Eqs. (80)-(82), it is easy to establish the relation

$$
\frac{\dot{w}}{1+w}=-3 H\left(c_{\mathrm{s}}^{2}-w\right)
$$

We now focus on the equation of state (83). For it, we obtain $w=k \rho_{\mathrm{b}}$ and $c_{\mathrm{eff}}^{2}=c_{\mathrm{s}}^{2}=2 w$. In that case, Eq. (116) reduces to

$\ddot{\delta}+2 H(3 w+1) \dot{\delta}-3 H^{2}\left(\frac{9 w^{2}}{2}+6 w+\frac{1}{2}\right) \delta-\frac{2 w c^{2}}{a^{2}} \Delta \delta=0$,

where we have used $\dot{w} / H=-3 w(1+w)$ according to Eq. (117). Measuring the evolution in terms of $a$ rather than in terms of $t$, and using the Friedmann Eqs. (81)-(82), we can rewrite Eq. (118) in the form

$a^{2} \frac{\mathrm{d}^{2} \delta}{\mathrm{d} a^{2}}+\frac{3}{2}(1+3 w) a \frac{\mathrm{d} \delta}{\mathrm{d} a}-\frac{3}{2}\left(9 w^{2}+12 w+1\right) \delta-\frac{2 w c^{2}}{H^{2} a^{2}} \Delta \delta=0$.

Finally, according to Eq. (88), we have

$w=\frac{ \pm 1}{R^{3} \mp 1}$

where the upper sign corresponds to $k>0$ and the lower to $k<0$. If, for simplicity, we ignore the Laplacian term in Eq. (119), which is permissible for large wavelengths, we obtain

$R^{2} \frac{\mathrm{d}^{2} \delta}{\mathrm{d} R^{2}}+\frac{3}{2}(1+3 w) R \frac{\mathrm{d} \delta}{\mathrm{d} R}-\frac{3}{2}\left(9 w^{2}+12 w+1\right) \delta=0$, where we have introduced the normalized radius $R=a / a_{*}$. For $R \rightarrow+\infty, w \rightarrow 0$ and Eq. (121) reduces to

$R^{2} \frac{\mathrm{d}^{2} \delta}{\mathrm{d} R^{2}}+\frac{3}{2} R \frac{\mathrm{d} \delta}{\mathrm{d} R}-\frac{3}{2} \delta=0$.

This returns the usual Eq. (55) of a pressureless fluid in the Einstein-de Sitter universe. Its solutions are given by Eq. (56).

We now consider the case of small $R$. For $k>0$, we have seen that the universe exists only for $R \geq 1$. Now, for $R \rightarrow 1$, $w \rightarrow+\infty$ so the differential Eq. (121) is ill-defined. This means that we must start the perturbation analysis at $t=t_{i}>0$. Harko (2011a) points out that the requirement $c_{\text {eff }}=c_{\mathrm{s}}<1$ (meaning that the velocity of sound must be lower than the velocity of light) implies $R>3^{1 / 3}$ and $w<1 / 2$. Therefore, we assume that the universe starts at $t_{*}$ such that $R\left(t_{*}\right)=3^{1 / 3}$. According to Eq. (92), we have

$\frac{3}{2} K t_{*}=\sqrt{2}-\arctan \sqrt{2} \simeq 0.4589$.

For $t \rightarrow t_{*}$, using $w \rightarrow 1 / 2$, Eq. (121) can be approximated by

$R^{2} \frac{\mathrm{d}^{2} \delta}{\mathrm{d} R^{2}}+\frac{15}{4} R \frac{\mathrm{d} \delta}{\mathrm{d} R}-\frac{111}{8} \delta=0$,

and its solutions are

$\delta_{+} \propto R^{\frac{-11+\sqrt{1009}}{8}}, \quad \delta_{-} \propto R^{\frac{-11-\sqrt{1009}}{8}}$.

The growth of perturbations is faster than in a cold EdS universe $\left(\delta_{+} \propto a^{2.6}\right.$ instead of $\left.a\right)$. On the other hand, for $k<0$, the universe starts at $R=0$ for $t \rightarrow-\infty$ implying $w=-1$. In that case, Eq. (121) can be approximated by

$R^{2} \frac{\mathrm{d}^{2} \delta}{\mathrm{d} R^{2}}-3 R \frac{\mathrm{d} \delta}{\mathrm{d} R}+3 \delta=0$,

and its solutions are

$\delta_{+} \propto R^{3}, \quad \delta_{-} \propto R$.

In contrast to a classical cold universe, the two solutions of the differential Eq. (126) are growing. Furthermore, the growth of perturbations is faster than in an EdS universe $\left(\delta_{+} \propto a^{3}\right.$ instead of $a$ ). Combining Eq. (127) with Eq. (95), we get

$\delta_{+}(t) \propto \mathrm{e}^{3 K t}, \quad \delta_{-}(t) \propto \mathrm{e}^{K t}$,

for $t \rightarrow-\infty$.

These simple estimates suggest that the growth of perturbations is faster in a BEC universe than in a pressureless (EdS) universe. This conclusion has previously been reached by Harko (2011a) for $k>0$ based on different equations. Our approach confirms this result and extends it to $k<0$. To be more precise, we have numerically solved Eq. (121) with initial condition $a_{i} / a_{0}=0.1, \delta_{i}=10^{-5}$ and $\delta_{i}^{\prime}=0$. The results are reported in Figs. 14 and 15. For $k>0$, we see that the perturbations grow more rapidly in a BEC universe than in the EdS universe as stated by Harko (2011a) ${ }^{13}$. For $k<0$, the situation is more difficult to analyze because the density contrast $\delta(a)$ changes sign when $q$ becomes significantly negative, and its evolution is not

13 In fact, his Figs. 3 and 4 seem to show the contrary (if we look at the behavior of the curves at the origin, we realize that the labels of the curves are reversed). This may be because the perturbations are initiated at different values of $a_{i}$, depending on $q$, while in our Fig. 14, on the contrary, the perturbations are initiated at the same value of $a_{i}$, for all $q$ 's. 


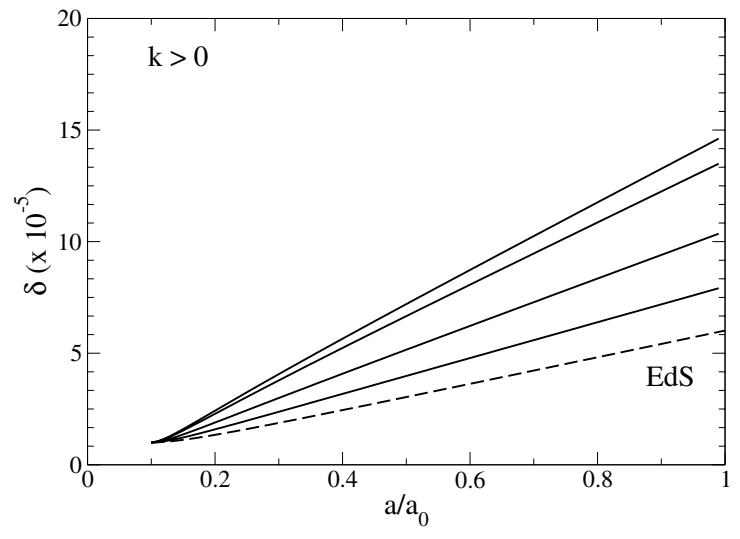

Fig. 14. Growth of perturbations in a BEC universe with $k>0$. We have numerically solved Eq. (121) with initial condition $a_{i} / a_{0}=0.1, \delta_{i}=$ $10^{-5}$ and $\delta_{i}^{\prime}=0$. We recall that $a / a_{0}=q^{1 / 3} R$. The condition $a\left(t_{*}\right) / a_{0}=$ $(3 q)^{1 / 3}<a_{i} / a_{0}=0.1$ imposes $q<3.33 \times 10^{-4}$. From bottom to top: $q=0.0001,0.0002,0.0003,0.00033$. The lowest curve corresponds to the EdS universe $(q=0)$.

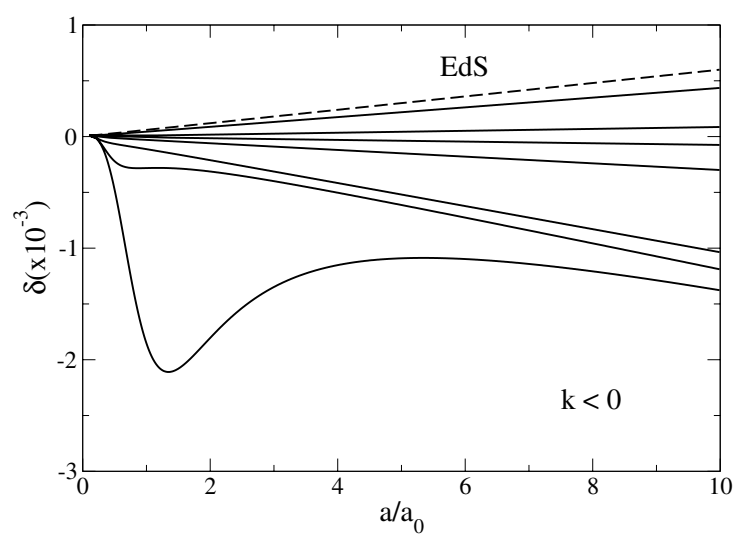

Fig. 15. Growth of perturbations in a BEC universe with $k<0$. We have numerically solved Eq. (121) with initial condition $a_{i} / a_{0}=0.1$, $\delta_{i}=10^{-5}$ and $\delta_{i}^{\prime}=0$. We recall that $a / a_{0}=(-q)^{1 / 3} R$. From top to bottom: $q=-0.0001,-0.0004,-0.0006,-0.001,-0.01,-0.1,-1$. The highest curve corresponds to the EdS universe $(q=0)$.

even monotonic. Furthermore, the BEC universe with $k<0$ behaves very differently from the EdS universe for small radii so the comparison between these two models is difficult to make. Finally, the Laplacian term in Eq. (118) has been neglected in our simple analysis. Now, we have seen in Sect. 6.2 that it is precisely this term that leads to an increase in the maximum growth rate in the (static) Jeans problem for a BEC with $k<0$. It is also this term that produces the rapid increase in density contrast of a BEC with $k<0$ in an expanding, nonrelativistic, EdS universe (see Fig. 6 of Sect. 7.2). Therefore, including this term in our analysis should enhance the growth of perturbations in the case of an attractive self-interaction.

\section{Dark fluid with a generalized equation of state}

\subsection{Linear equation of state}

One possibility for understanding the present-day acceleration in the expansion of the universe is to invoke a form of dark energy arising from a non-zero value of the cosmological constant $\Lambda$ (see Sect. 8.4). However, the physical meaning of $\Lambda$ is not clearly understood. A major problem is that most quantum field theories predict a huge cosmological constant from the energy of the quantum vacuum, that is more than 100 orders of magnitude too high. Therefore, other approaches have been developed to understand the acceleration of the universe without invoking the cosmological constant. One possibility is to consider a "dark fluid" with a negative pressure. In this context, many workers (see the review of Peebles \& Ratra 2003) have considered a linear equation of state

$p=\alpha \rho c^{2}$

with $-1 \leq \alpha \leq 1$. For this equation of state, the Friedmann Eqs. (80)-(82) with $\kappa=\Lambda=0$ reduce to

$\frac{\mathrm{d} \rho_{\mathrm{b}}}{\mathrm{d} t}+3 \frac{\dot{a}}{a}(1+\alpha) \rho_{\mathrm{b}}=0$,

$\frac{\ddot{a}}{a}=-\frac{4 \pi G}{3}(1+3 \alpha) \rho_{b}, \quad\left(\frac{\dot{a}}{a}\right)^{2}=\frac{8 \pi G}{3} \rho_{\mathrm{b}}$.

Equation (130) leads to the relation $\rho_{b} a^{3(1+\alpha)} \sim 1$. We note that the effect of a cosmological constant $\Lambda$ is equivalent to a fluid with an equation of state $p=-\rho c^{2}(\alpha=-1)$ since, in that case, $\rho_{\mathrm{b}}$ is constant and can be written $\rho_{\mathrm{b}}=\rho_{\Lambda} \equiv \Lambda / 8 \pi G$ (compare Eqs. (131-b) and (82)). This equation of state leads to an exponential growth of the scale factor

$a=a(0) \mathrm{e}^{\sqrt{\Lambda / 3} t}$.

On the other hand, for $-1<\alpha \leq 1$, Eqs. (130) and (131) generate a model of the form

$a \propto t^{2 /[3(1+\alpha)]}, \quad H=\frac{\dot{a}}{a}=\frac{2}{3(1+\alpha) t}$,
$\rho_{\mathrm{b}}=\frac{1}{6 \pi G(1+\alpha)^{2} t^{2}}$.

According to Eq. (131-a), this universe is accelerating for $-1<$ $\alpha<\alpha_{\mathrm{c}} \equiv-1 / 3$ and decelerating for $\alpha>\alpha_{\mathrm{c}}$. For $\alpha=\alpha_{\mathrm{c}}$, the scale factor increases linearly with time $(a \propto t)$. The EdS universe corresponds to $\alpha=0$. For the general model (133) $-(134)$, the age of the universe is $t_{0}=2 /\left[3(1+\alpha) H_{0}\right]$ where $H_{0}=$ $2.273 \times 10^{-18} \mathrm{~s}^{-1}$ is the current value of the Hubble constant. For the critical value $\alpha_{\mathrm{c}}=-1 / 3$, the age of the universe is $t_{0}=H_{0}^{-1}=13.95 \mathrm{Gyr}$ which is close to the value $13.75 \mathrm{Gyr}$ predicted by the $\Lambda$ CDM model. However, the deceleration parameter $Q=-\ddot{a} a / \dot{a}^{2}=(1+3 \alpha) / 2$ vanishes for $\alpha=-1 / 3$, while its current value is $\sim-0.5$. On the other hand, the linear equation of state (129) does not allow for a transition between a phase of deceleration and a phase of acceleration, while such a transition is likely in our universe. It may therefore be interesting to generalize this model.

\subsection{Generalized equation of state}

We consider a generalized equation of state in the form

$p=\left(\alpha \rho+k \rho^{2}\right) c^{2}$,

with $-1 \leq \alpha \leq 1$ and $k$ positive or negative (the case $\alpha=-1$ is specifically treated in Appendix C). In this approach, a single "dark fluid" combines the properties of a BEC dark matter described by the equation of state (83) and of a dark energy described by the equation of state (129). The equation of state of the BEC dark matter $\left(\propto \rho^{2}\right)$ dominates in the early universe where the density is high, while the dark energy $(\propto \rho)$ dominates in the present-day universe where the density is low. The equation of 
P. H. Chavanis: Growth of perturbations in an expanding universe with Bose-Einstein condensate dark matter

state of the BEC also dominates on the scale of dark matter halos where the density is high. This makes the study of this equation of state particularly interesting. Another nice feature of this equation of state is that it admits fully analytical solutions ${ }^{14}$.

For the equation of state (135), the Friedmann Eq. (80) becomes

$\frac{\mathrm{d} \rho_{\mathrm{b}}}{\mathrm{d} t}+3 \frac{\dot{a}}{a} \rho_{\mathrm{b}}\left(1+\alpha+k \rho_{\mathrm{b}}\right)=0$.

This equation can be integrated into

$\rho_{\mathrm{b}}=\frac{A(1+\alpha)}{a^{3(1+\alpha)}-k A}$,

where $A>0$ is a constant. For $a \rightarrow+\infty, \rho_{\mathrm{b}} \sim A(1+\alpha) / a^{3(1+\alpha)}$. When $k>0$, the density exists only for $a>a_{*}=(k A)^{1 /[3(1+\alpha)]}$. For $a \rightarrow a_{*}, \rho_{\mathrm{b}} \rightarrow+\infty$. When $k<0$, the density is defined for all $a$ and has a finite value $\rho_{\mathrm{b}}=(1+\alpha) /|k|$ when $a \rightarrow 0$.

Combining Eqs. (81) and (135), and taking $\Lambda=0$, we obtain

$\frac{\ddot{a}}{a}=-\frac{4 \pi G \rho_{\mathrm{b}}}{3}\left(1+3 \alpha+3 k \rho_{\mathrm{b}}\right)$.

We then define a critical density and a critical scale factor

$\rho_{\mathrm{c}}=-\frac{1+3 \alpha}{3 k}, \quad a_{\mathrm{c}}=\left(\frac{-2 k A}{1+3 \alpha}\right)^{\frac{1}{3(1+\alpha)}}$,

corresponding to a possible inflexion point $(\ddot{a}=0)$ in the curve $a(t)$. When $k>0$ and $\alpha \geq-1 / 3$, the universe is always decelerating $(\ddot{a}<0)$. When $k>0$ and $\alpha<-1 / 3$, the universe is decelerating for $\rho_{\mathrm{b}}>\rho_{\mathrm{c}}$ (i.e. $a<a_{\mathrm{c}}$ ) and accelerating for $\rho_{\mathrm{b}}<\rho_{\mathrm{c}}$ (i.e. $a>a_{\mathrm{c}}$ ). When $k<0$ and $\alpha \leq-1 / 3$, the universe is always accelerating $(\ddot{a}>0)$. When $k<0$ and $\alpha>-1 / 3$, the universe is accelerating for $\rho_{\mathrm{b}}>\rho_{\mathrm{c}}$ (i.e. $a<a_{\mathrm{c}}$ ) and decelerating for $\rho_{\mathrm{b}}<\rho_{\mathrm{c}}$ (i.e. $a>a_{\mathrm{c}}$ ).

To determine the temporal evolution of $a(t)$, we assume that the universe is flat $(\kappa=0)$. Combining Eqs. (82) and (137), we get

$\dot{a}=\left[\frac{8 \pi G A}{3}(1+\alpha)\right]^{1 / 2} \frac{a}{\sqrt{a^{3(1+\alpha)}-k A}}$.

For $a \rightarrow+\infty$, we recover the solution (133)-(134). It is convenient to define $a_{*}=(|k| A)^{1 /[3(1+\alpha)]}$ and introduce $R=a / a_{*}$. In that case, the density is given by

$\rho_{\mathrm{b}}=\frac{1+\alpha}{|k|} \frac{1}{R^{3(1+\alpha)} \mp 1}$,

and Eq. (140) can be rewritten

$\dot{R}=\frac{K R}{\sqrt{R^{3(1+\alpha)} \mp 1}}$,

where the upper sign - corresponds to $k>0$ and the lower sign + corresponds to $k<0$. On the other hand, we have defined the constant

$K=\left[\frac{8 \pi G}{3|k|}(1+\alpha)\right]^{1 / 2}$.

\footnotetext{
${ }^{14}$ Equation (135) can be written as $p_{\mathrm{b}}=w(t) \rho_{\mathrm{b}} c^{2}$ where $w(t)$ is a function of time. Many authors have considered an equation of state of that form with some prescribed function $w(t)$. In our model, $w(t)$ is not an ad hoc function but is explicitly given by $w(t)=\alpha+k \rho_{\mathrm{b}}(t)$.
}

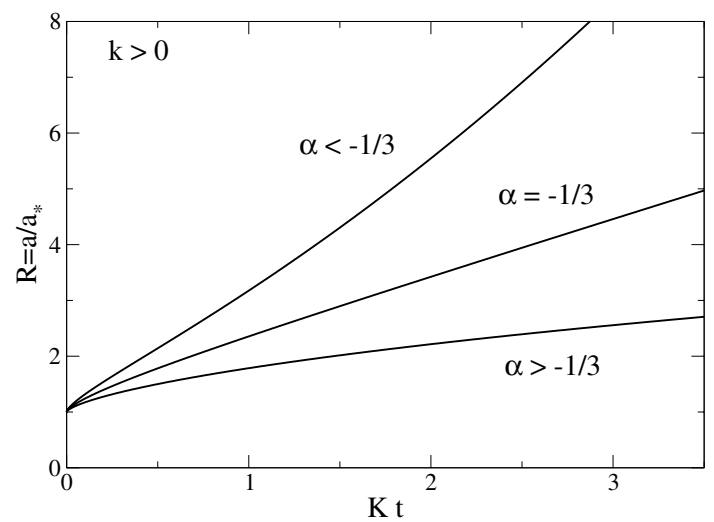

Fig. 16. Evolution of the scale factor in the case $k>0$ for different values of $\alpha$ (specifically $\alpha=-2 / 3, \alpha=-1 / 3$, and $\alpha=1 / 2$ ).

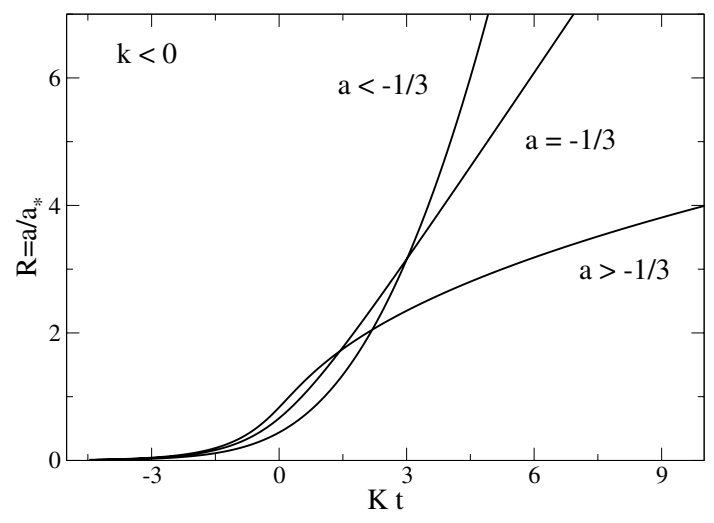

Fig. 17. Evolution of the scale factor in the case $k<0$ for different values of $\alpha$ (specifically $\alpha=-2 / 3, \alpha=-1 / 3$, and $\alpha=1 / 2$ ).

For $k>0$, the solution of Eq. (142) is

$\sqrt{R^{3(1+\alpha)}-1}-\arctan \sqrt{R^{3(1+\alpha)}-1}=\frac{3(1+\alpha)}{2} K t$,

where the constant of integration has been set equal to zero. In this model, the universe starts at a finite time $t=0$ (see Sect. 9.4 for a revision of this statement) with a finite radius $R(0)=1$ and an infinite density $\rho_{\mathrm{b}}(0)=\infty$. For $t \rightarrow 0, R \simeq 1+[3 /(4(1+$ $\alpha))]^{1 / 3}(K t)^{2 / 3}$. For $t \rightarrow+\infty$, it asymptotically approaches the solution (133)-(134); i.e., $R \sim(3(1+\alpha) K t / 2)^{2 /[3(1+\alpha)]}$. When $\alpha \geq-1 / 3$, the universe is always decelerating $(\ddot{R}<0)$. When $\alpha<-1 / 3$, the universe is decelerating for $\rho_{\mathrm{b}}>\rho_{\mathrm{c}}$ (i.e. $R<R_{\mathrm{c}}^{+} \equiv$ $\left.(-2 /(1+3 \alpha))^{1 /[3(1+\alpha)]}\right)$ and accelerating for $\rho_{\mathrm{b}}<\rho_{\mathrm{c}}\left(\right.$ i.e. $\left.R>R_{\mathrm{c}}^{+}\right)$. The time $t_{\mathrm{c}}$ at which the universe starts accelerating is given by

$\frac{3(1+\alpha)}{2} K t_{\mathrm{c}}=\sqrt{\frac{-3(1+\alpha)}{1+3 \alpha}}-\arctan \sqrt{\frac{-3(1+\alpha)}{1+3 \alpha}}$.

Some possible evolutions of $R(t)$ corresponding to $\alpha<-1 / 3$, $\alpha=1 / 3$, and $\alpha>1 / 3$ are represented in Fig. 16 .

For $k<0$, the solution of Eq. (142) is

$$
\sqrt{R^{3(1+\alpha)}+1}-\ln \left(\frac{1+\sqrt{R^{3(1+\alpha)}+1}}{R^{3(1+\alpha) / 2}}\right)=\frac{3(1+\alpha)}{2} K t,
$$

where the constant of integration has been set to zero. In this model, the universe starts from $t \rightarrow-\infty$ with a vanishing radius $R(-\infty)=0$ and a finite density $\rho_{\mathrm{b}}(-\infty)=(1+\alpha) /|k|$. For $t \rightarrow$ $+\infty$, it asymptotically approaches the solution (133)-(134) i.e. $R \sim(3(1+\alpha) K t / 2)^{2 /[3(1+\alpha)]}$. When $\alpha \leq-1 / 3$, the universe is 
always accelerating ( $\ddot{a}>0$ ). When $\alpha>-1 / 3$, the universe is accelerating for $\rho_{\mathrm{b}}>\rho_{\mathrm{c}}$ (i.e. $\left.R<R_{\mathrm{c}}^{-} \equiv(2 /(1+3 \alpha))^{1 /[3(1+\alpha)]}\right)$ and decelerating for $\rho_{\mathrm{b}}<\rho_{\mathrm{c}}$ (i.e. $R>R_{\mathrm{c}}^{-}$). The time $t_{\mathrm{c}}$ at which the universe starts decelerating is given by

$$
\frac{3(1+\alpha)}{2} K t_{\mathrm{c}}=\sqrt{\frac{3(1+\alpha)}{1+3 \alpha}}-\ln \left(\frac{\sqrt{1+3 \alpha}+\sqrt{3(1+\alpha)}}{\sqrt{2}}\right) \text {. }
$$

For $t \rightarrow-\infty, R \rightarrow 0$ and the asymptotic expansion of Eq. (146) yields

$R(t) \propto \mathrm{e}^{K t}$.

Some possible evolutions of $R(t)$ corresponding to $\alpha<-1 / 3$, $\alpha=1 / 3$, and $\alpha>1 / 3$ are represented in Fig. 17 .

\subsection{Optimal parameters}

We can easily extend the analysis of Sect. 8.3 to the equation of state (135). The density can be written

$\rho_{\mathrm{b}}=\rho_{0} \frac{1-q}{\left(\frac{a}{a_{0}}\right)^{3(1+\alpha)}-q}$,

where

$q=\frac{k \rho_{0}}{1+\alpha+k \rho_{0}}$

implying

$k \rho_{0}=\frac{q(1+\alpha)}{1-q}$.

We note that $q$ is positive for $k>0$ and negative for $k<0$. Furthermore, $q=0$ for $k=0, q \rightarrow 1$ for $k \rightarrow+\infty$ and $q \rightarrow-\infty$ for $k \rightarrow-(1+\alpha) / \rho_{0}$. Using these results, the Friedmann Eq. (82) giving the temporal evolution of the scale factor can be rewritten (for $\kappa=\Lambda=0$ ):

$\dot{a}=H_{0} \sqrt{\Omega_{0}}(1-q)^{1 / 2} \frac{a}{\sqrt{\left(\frac{a}{a_{0}}\right)^{3(1+\alpha)}-q}}$.

We can make the connection with the previous notations by setting

$a_{*}=a_{0}|q|^{1 /[3(1+\alpha)]}, \quad K=H_{0} \sqrt{\Omega_{0}}\left(\frac{1-q}{|q|}\right)^{1 / 2}$.

In that case, Eq. (152) takes the form of Eq. (142) and its solutions are given by Eqs. (144) and (146). Returning to the notations of this section, we find that the evolution of $a(t)$ is given by Eqs. (104)-(106) where all three are replaced by $3(1+\alpha)$. The other equations can be easily generalized.

We assume that all the content of the universe is in the dark fluid with the generalized equation of state (135) so that $\Omega_{0}=1$. We call $t_{0}$ the time at which $a / a_{0}=1$. For $0<q<1$,

$H_{0} t_{0}=\frac{2}{3(1+\alpha)}\left\{1-\sqrt{\frac{q}{1-q}} \arctan \sqrt{\frac{1-q}{q}}\right\}$,

for $q<0$,

$H_{0} t_{0}=\frac{2}{3(1+\alpha)}\left\{1-\sqrt{\frac{-q}{1-q}} \ln (\sqrt{-q}+\sqrt{1-q})\right\}$,

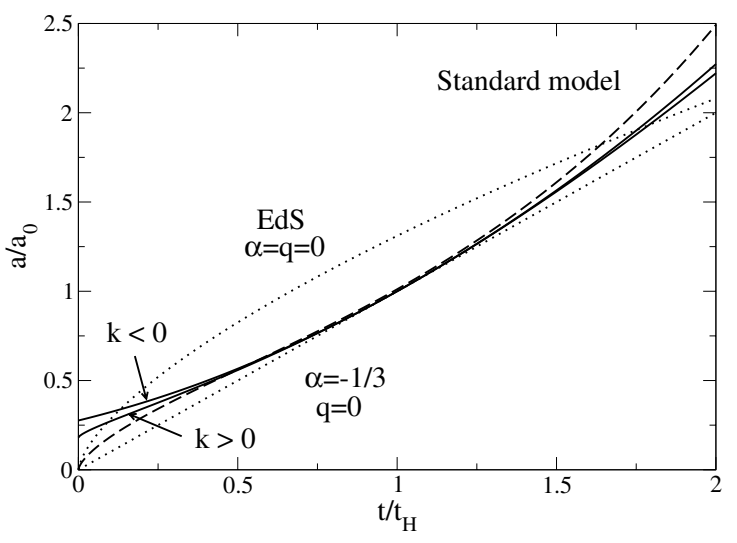

Fig. 18. Evolution of the scale factor $a(t)$ as a function of time. We have represented the standard $\Lambda \mathrm{CDM}$ model (long-dashed line) and a dark fluid (solid lines) described by an equation of state $p=\left(\alpha \rho+k \rho^{2}\right) c^{2}$ with optimal parameters $(q=0.303471, \alpha=-0.76782367)$ and $(q=-0.383589, \alpha=-0.53880367)$. We have also represented the EdS universe $p=0$ (upper dotted line) and the universe described by a linear equation of state $p=-\rho c^{2} / 3$ (lower dotted line).

and for $q=0$,

$H_{0} t_{0}=\frac{2}{3(1+\alpha)}$.

These expressions basically give the age of the universe $t_{0}$ as a function of $q$ and $\alpha$.

The deceleration parameter is defined by

$Q=-\frac{\ddot{a} a}{\dot{a}^{2}}=\frac{1+3 w(t)}{2}$,

where $w(t) \equiv p_{\mathrm{b}} / \rho_{\mathrm{b}} c^{2}$. For the equation of state (135), $w(t)=$ $\alpha+k \rho_{\mathrm{b}}(t)$. Using Eq. (151), we find that the present-day value of the deceleration parameter can be expressed in our model as

$Q_{0}=\frac{3 \alpha+2 q+1}{2(1-q)}$.

The observed value of $Q_{0}$ is close to -0.5 . If we take $Q_{0}=-1 / 2$, Eq. (158) yields $\alpha=-(q+2) / 3$. On the other hand, the age of the universe is about $13.75 \mathrm{Gyr}$ in the standard model. If we take $t_{0}=H_{0}^{-1}$ (which is close to this value) and use the previous relation between $\alpha$ and $q$, Eqs. (154) and (155) determine the optimal values of $q$ and $\alpha$. For $0<q<1$, the optimal value of $q$ is given by

$\frac{1+q}{2}-\sqrt{\frac{q}{1-q}} \arctan \sqrt{\frac{1-q}{q}}=0$.

We find $q=0.303471$ and $\alpha=-0.76782367$. For $q<0$, the optimal value of $q$ is given by

$\frac{1+q}{2}-\sqrt{\frac{-q}{1-q}} \ln (\sqrt{-q}+\sqrt{1-q})=0$.

We find $q=-0.383589$ and $\alpha=-0.53880367$.

The evolution of the scale factor $a(t)$ in a universe filled with a dark fluid with equation of state (135) is represented in Fig. 18 for the optimal values of $(q, \alpha)$ obtained previously. It gives a relatively good agreement with the standard $\Lambda$ CDM model based on a nonvanishing value of the cosmological constant (see Figs. 12 and 13). By construction, these three curves give the same values of the age of the universe $t_{0} \sim H_{0}^{-1}$ and of 
the present-day deceleration parameter $Q_{0} \sim-0.5$. The model with $k>0$ is decelerating then accelerating. Using Eqs. (145) and (153), we find that the acceleration starts at $H_{0} t_{c} \sim 0.2$ which is earlier than the value $H_{0} t_{\mathrm{c}} \sim 0.5$ predicted by the standard $\Lambda$ CDM model. The model with $k<0$ is always accelerating. We have also represented the model corresponding to a linear equation of state $(q=0)$ with $\alpha=-1 / 3$ yielding $a / a_{0}=H_{0} t$. It gives the correct age of the universe but has a vanishing deceleration parameter. Finally, we have represented the (pressureless) EdS model corresponding to $\alpha=q=0$ yielding $a / a_{0}=\left(3 H_{0} t / 2\right)^{2 / 3}$. It does not give the correct age of the universe and is decelerating instead of accelerating. The other models with $\alpha=0$ and $q \neq 0$ do not do better. This shows that we must combine the two equations of state $\alpha \rho$ and $k \rho^{2}$ to obtain a good agreement with the standard $\Lambda \mathrm{CDM}$ model, i.e. we need an equation of state with two parameters.

\subsection{Theory of linear perturbations}

In this section, we extend the theory of linear perturbations developed in Sect. 8.5 to the case of the generalized equation of state (135). For this equation of state, $w=\alpha+k \rho_{\mathrm{b}}$ and $c_{\mathrm{eff}}^{2}=c_{\mathrm{s}}^{2}=2 w-\alpha$. According to Eq. (141), we have

$w=\alpha \pm \frac{1+\alpha}{R^{3(1+\alpha)} \mp 1}$,

where the upper sign corresponds to $k>0$ and the lower sign corresponds to $k<0$. The generalization of Eq. (121) is

$$
\begin{aligned}
R^{2} \frac{\mathrm{d}^{2} \delta}{\mathrm{d} R^{2}} & +\frac{3}{2}(1+3 w-4 \alpha) R \frac{\mathrm{d} \delta}{\mathrm{d} R} \\
& -\frac{3}{2}\left(9 w^{2}+12 w+1-8 \alpha-6 \alpha^{2}\right) \delta=0 .
\end{aligned}
$$

For $R \rightarrow+\infty, w \rightarrow \alpha$ and Eq. (162) reduces to

$R^{2} \frac{\mathrm{d}^{2} \delta}{\mathrm{d} R^{2}}+\frac{3}{2}(1-\alpha) R \frac{\mathrm{d} \delta}{\mathrm{d} R}-\frac{3}{2}(\alpha+1)(3 \alpha+1) \delta=0$.

This is the equation for the density contrast corresponding to the linear equation of state (129). Its solutions are

$$
\delta_{+} \propto R^{1+3 \alpha}, \quad \delta_{-} \propto R^{-\frac{3}{2}(1+\alpha)} .
$$

Now we consider the case of small $R$. For $k>0$, the requirement $c_{\mathrm{eff}}=c_{\mathrm{S}}<1$ implies $R>[(3+\alpha) /(1-\alpha)]^{1 /[3(1+\alpha)]}$ and $w<$ $(\alpha+1) / 2$. Therefore, we assume that the universe starts at $t_{*}$ such that $R\left(t_{*}\right)=[(3+\alpha) /(1-\alpha)]^{1 /[3(1+\alpha)]}$. According to Eq. (144), we have

$$
\frac{3(1+\alpha)}{2} K t_{*}=\sqrt{\frac{2(1+\alpha)}{1-\alpha}}-\arctan \sqrt{\frac{2(1+\alpha)}{1-\alpha}} .
$$

For $t \rightarrow t_{*}$, using $w \rightarrow(\alpha+1) / 2$, Eq. (162) can be approximated by

$R^{2} \frac{\mathrm{d}^{2} \delta}{\mathrm{d} R^{2}}+\frac{15}{4}(1-\alpha) R \frac{\mathrm{d} \delta}{\mathrm{d} R}+\frac{3}{8}\left(15 \alpha^{2}-10 \alpha-37\right) \delta=0$,

and its solutions are

$\delta_{ \pm} \propto R^{\frac{-11+15 \alpha \pm \sqrt{-135 \alpha^{2}-90 \alpha+1009}}{8}}$.

For $k<0$, the universe starts at $R=0$ for $t \rightarrow-\infty$ implying $w=-1$. In that case, Eq. (162) can be approximated by

$R^{2} \frac{\mathrm{d}^{2} \delta}{\mathrm{d} R^{2}}-3(1+2 \alpha) R \frac{\mathrm{d} \delta}{\mathrm{d} R}+3(1+\alpha)(1+3 \alpha) \delta=0$,

and its solutions are

$\delta_{+} \propto R^{3(1+\alpha)}, \quad \delta_{-} \propto R^{1+3 \alpha}$.

\section{Conclusion}

Following the proposal of Böhmer \& Harko (2007) and others (see a short review in Chavanis 2011a), we considered the possibility that the dark matter in the universe is a self-gravitating BEC with short-range interactions, and we theoretically explored the consequences of this hypothesis. For the sake of generality, we considered the case of positive and negative scattering lengths.

At the level of dark matter halos, a positive scattering length, equivalent to a repulsive self-interaction generating a positive pressure, is able to stabilize the halos with respect to gravitational collapse. This leads to dark matter halos without density cusps, which are equivalent to polytropes of index $n=1$ (more generally, the barotropic equation of state is fixed by the form of the self-interaction so that other configurations are possible). Alternatively, if the scattering length is negative, equivalent to an attractive self-interaction generating negative pressure, the dark matter is very unstable and collapses above a very low critical mass $M_{\max }=1.012 \hbar / \sqrt{\left|a_{\mathrm{s}}\right| G m}$ (Chavanis 2011a). When these ideas are applied to an infinite homogeneous cosmic fluid (Jeans problem), it is found that a negative scattering length can increase the maximum growth rate of the instability and accelerate the formation of structures. The virtues of these results could be combined by assuming that the scattering length changes sign in the course of the evolution. It could initially be negative to help form structures and become positive (due to a change of density, magnetic fields, radiation etc.) to prevent complete gravitational collapse. The mechanism of this change in sign is, however, unknown so that this idea remains highly speculative. However, some terrestrial experiments have demonstrated that certain atoms can have negative scattering lengths, that their scattering length can depend on the magnetic field, and that it is possible in principle to manipulate the value and the sign of $a_{\mathrm{s}}$ (Fedichev et al. 1996). Therefore, a BEC is a serious candidate for having positive and/or negative pressure (see Appendix B for further remarks about the values of the BEC parameters).

At the cosmological level, we constructed models of universe composed of BEC dark matter with attractive or repulsive self-interaction. We first studied the academic situation where the universe is only made of BEC dark matter. A BEC universe with positive scattering length, leading to positive pressure, is not qualitatively very different from a classical Einstein-de Sitter universe. It also emerges at a primordial time $t=0$ from a bigbang singularity where the density is infinite, and undergoes a decelerating expansion asymptotically equivalent to the EdS universe. A difference, however, is that the initial scale factor $a(0)$ is finite. On the other hand, a BEC universe with negative scattering length, leading to negative pressure, differs markedly from previous models. It starts from $t \rightarrow-\infty$ with a vanishing radius and a finite density, has an initial accelerating expansion then decelerates, and asymptotically behaves like the EdS universe. This model universe exists for any time in the past, and there is no bigbang singularity. When we add the effects of radiation, baryonic matter, and dark energy (via the cosmological constant), the picture is different. In that case, a BEC universe with attractive or repulsive self-interaction starts from a singularity at $t=0$ where the density is infinite. It first experiences a phase of decelerating expansion followed by a phase of accelerating expansion. For $k \rightarrow 0$ we recover the standard $\Lambda$ CDM model, but for $k \neq 0$, the evolution of the scale factor in a BEC universe can be substantially different. The model with $k>0$ expands more rapidly than the standard model $(k=0)$. The initial scale factor is finite $(a(0)>0)$ and the BEC energy contribution dominates the 
radiation and the other cosmological energy terms, leading to a decelerating expansion at the beginning. The model with $k<0$ expands less rapidly than the standard model. The initial scale factor vanishes $(a(0)=0)$ and the radiation dominates the BEC energy and the other cosmological energy terms, leading also to a decelerating expansion at the beginning. In both models, the dark energy dominates at late times, leading to an accelerating expansion at the present day. Finally, we considered a "dark fluid" with generalized equation of state $p=\left(\alpha \rho+k \rho^{2}\right) c^{2}$ having a component $p=k \rho^{2} c^{2}$ similar to a BEC dark matter and a component $p=\alpha \rho c^{2}$ mimicking the effect of the cosmological constant (dark energy). We found optimal parameters $(\alpha, k)$ that give good agreement with the standard $\Lambda$ CDM model. We studied the growth of perturbations in these different models and confirmed the previous claim of Harko (2011a) that the density contrast increases more rapidly in a BEC universe than in the standard $\Lambda$ CDM model.

The idea that dark matter could be a BEC is fascinating and probably deserves further research.

Acknowledgements. I am grateful to E. Sansing for some useful advice.

\section{Appendix A: Solution of the differential Eq. (58)}

In this Appendix, we provide some analytical solutions of the differential Eq. (58). If we define

$\mu=\frac{3 k^{4}}{2 \kappa_{Q}^{4}}, \quad \lambda=\frac{3 k^{2}}{2 \kappa_{\mathrm{J}}^{2}}, \quad \alpha=\frac{4-3 \gamma}{2}$,

this equation can be written as

$\frac{\mathrm{d}^{2} f}{\mathrm{~d} a^{2}}-\frac{1}{2 a} \frac{\mathrm{d} f}{\mathrm{~d} a}+\frac{1}{a^{2}}\left(\frac{\mu}{a}+\lambda a^{2 \alpha}-1\right) f=0$.

With the change in function $f(a)=a^{3 / 4} g(a)$, we get

$\frac{\mathrm{d}^{2} g}{\mathrm{~d} a^{2}}+\frac{1}{a} \frac{\mathrm{d} g}{\mathrm{~d} a}+\frac{1}{a^{2}}\left(\frac{\mu}{a}+\lambda a^{2 \alpha}-\frac{25}{16}\right) g=0$.

Assuming $\alpha \neq 0$ and defining $x=a^{\alpha}$, we obtain

$x^{2} \frac{\mathrm{d}^{2} g}{\mathrm{~d} x^{2}}+x \frac{\mathrm{d} g}{\mathrm{~d} x}+\frac{1}{\alpha^{2}}\left(\frac{\mu}{x^{1 / \alpha}}+\lambda x^{2}-\frac{25}{16}\right) g=0$.

We have not been able to find the general solution of this differential equation in terms of simple functions. We therefore consider particular cases. We first consider the case $\mu=0$. Then

$x^{2} \frac{\mathrm{d}^{2} g}{\mathrm{~d} x^{2}}+x \frac{\mathrm{d} g}{\mathrm{~d} x}+\frac{1}{\alpha^{2}}\left(\lambda x^{2}-\frac{25}{16}\right) g=0$

For $\lambda>0$, defining $y=\sqrt{\lambda} x / \alpha$ and $p=5 / 4 \alpha$, the foregoing equation becomes

$y^{2} \frac{\mathrm{d}^{2} g}{\mathrm{~d} y^{2}}+y \frac{\mathrm{d} g}{\mathrm{~d} y}+\left(y^{2}-p^{2}\right) g=0$.

This is a Bessel equation whose regular solutions are $g=J_{ \pm p}(y)$. Coming back to the original variables, we get

$f(a) \propto a^{3 / 4} J_{ \pm \frac{5}{4 \alpha}}\left(\frac{\sqrt{\lambda} a^{\alpha}}{\alpha}\right)$.
We recall the asymptotic expansions of the Bessel functions

$J_{v}(z) \sim \sqrt{\frac{2}{\pi z}} \cos \left(z-\frac{1}{2} v \pi-\frac{1}{4} \pi\right), \quad(z \rightarrow+\infty)$,

$J_{v}(z)=\left(\frac{1}{2} z\right)^{v} \sum_{k=0}^{+\infty} \frac{\left(-\frac{1}{4} z^{2}\right)^{k}}{k ! \Gamma(v+k+1)}, \quad(z \rightarrow 0)$.

For $\lambda<0$, defining $y=\sqrt{-\lambda} x / \alpha$ and $p=5 / 4 \alpha$, Eq. (A.5) becomes

$y^{2} \frac{\mathrm{d}^{2} g}{\mathrm{~d} y^{2}}+y \frac{\mathrm{d} g}{\mathrm{~d} y}-\left(y^{2}+p^{2}\right) g=0$.

This is a Bessel equation whose regular solutions are $g=I_{ \pm p}(y)$. Coming back to the original variables, we get

$f(a) \propto a^{3 / 4} I_{ \pm \frac{5}{4 \alpha}}\left(\frac{\sqrt{-\lambda} a^{\alpha}}{\alpha}\right)$.

We recall the asymptotic expansions of the modified Bessel functions

$I_{\nu}(z) \sim \frac{e^{z}}{\sqrt{2 \pi z}}\left(1-\frac{4 v^{2}-1}{8 z}+\ldots\right), \quad(z \rightarrow+\infty)$,

$I_{v}(z)=\left(\frac{1}{2} z\right)^{v} \sum_{k=0}^{+\infty} \frac{\left(\frac{1}{4} z^{2}\right)^{k}}{k ! \Gamma(v+k+1)}, \quad(z \rightarrow 0)$.

For $\alpha=0$ and $\mu=0$, Eq. (A.2) becomes

$\frac{\mathrm{d}^{2} f}{\mathrm{~d} a^{2}}-\frac{1}{2 a} \frac{\mathrm{d} f}{\mathrm{~d} a}+\frac{1}{a^{2}}(\lambda-1) f=0$.

Looking for solutions of the form $f(a) \propto a^{n}$ we find that

$f(a) \propto a^{\frac{3}{4} \pm \sqrt{\frac{25}{16}-\lambda}}$.

Finally, for $\alpha=0$ and $\mu \neq 0$, Eq. (A.2) can be rewritten as

$\frac{\mathrm{d}^{2} f}{\mathrm{~d} a^{2}}-\frac{1}{2 a} \frac{\mathrm{d} f}{\mathrm{~d} a}+\frac{1}{a^{2}}\left(\frac{\mu}{a}+\lambda-1\right) f=0$.

Proceeding as before, we find that the solution of this equation is

$f(a) \propto a^{3 / 4} J_{ \pm \frac{1}{2} \sqrt{25-16 \lambda}}\left(2 \sqrt{\frac{\mu}{a}}\right)$.

\section{Appendix B: Estimate of the BEC parameters}

In this Appendix, we derive some constraints on the BEC parameters. A complementary analysis is made in Appendix A of Chavanis \& Delfini (2011). The parameter $k$ appearing in the equation of state (83) of a BEC is defined by $k=2 \pi a_{\mathrm{s}} \hbar^{2} / \mathrm{m}^{3} c^{2}$ and the present-day critical density is $\left(\rho_{\mathrm{c}}\right)_{0}=3 H_{0}^{2} / 8 \pi G$. Introducing the dimensionless parameter $\lambda / 8 \pi=a_{\mathrm{s}} m c / \hbar$ (see Chavanis 2011a), we obtain the general relations

$$
\begin{aligned}
k\left(\rho_{\mathrm{c}}\right)_{0} & =\frac{3}{4}\left(H_{0} t_{\mathrm{P}}\right)^{2}\left(\frac{M_{\mathrm{P}}}{m}\right)^{4} \frac{\lambda}{8 \pi} \\
& =9.776 \times 10^{-12}\left(\frac{1 \mathrm{eV} / c^{2}}{m}\right)^{4} \lambda,
\end{aligned}
$$

and

$\frac{\lambda}{8 \pi}=5.068 \times 10^{-9} \frac{a_{\mathrm{s}}}{1 \mathrm{fm}} \frac{m}{1 \mathrm{eV} / c^{2}}$. 
On the other hand, the radius of a BEC dark matter halo is given by (Arbey et al. 2003; Böhmer \& Harko 2007; Chavanis 2011a):

$R=\pi\left(\frac{a_{\mathrm{s}} \hbar^{2}}{G m^{3}}\right)^{1 / 2}=\pi\left(\frac{\lambda \hbar^{3}}{8 \pi G c}\right)^{1 / 2} \frac{1}{m^{2}}$,

so that

$$
\left(\frac{R}{1 \mathrm{kpc}}\right)^{2}=2.394 \times 10^{3}\left(\frac{1 \mathrm{eV} / c^{2}}{m}\right)^{4} \lambda .
$$

Therefore, the typical size of the dark matter halos determines the ratio $\lambda / \mathrm{m}^{4}$. Taking $R=10 \mathrm{kpc}$, we obtain $\left(m /\left(\mathrm{eV} / \mathrm{c}^{2}\right)\right) / \lambda^{1 / 4}=2.21$. Now, we notice that the parameter $k\left(\rho_{\mathrm{c}}\right)_{0}$, given by Eq. (B.1), only depends on $\lambda / m^{4}$. Using the previous estimate, we get $k\left(\rho_{\mathrm{c}}\right)_{0}=4.08 \times 10^{-13}$. We conclude that the dimensionless parameter $q$, defined by Eq. (98), is very small.

This estimate, which does not rely on any free parameter, seems to indicate that the pressure of the BEC dark matter is totally negligible on the scale of the cosmic fluid (since $q \simeq 0$ like in a pressureless universe), while it is important on the scale of galactic halos (since $R^{2} \propto \lambda / \mathrm{m}^{4}$ ). This seems to be bad news for the BEC cosmology. In fact, this result implies either that (i) the BEC dark matter can be treated as a pressureless fluid, like the ordinary dark matter, on the cosmological scale (i.e. in the Friedmann equations) or that (ii) the BEC parameters (in particular the scattering length $a_{\mathrm{s}}$ ) are different in the homogeneous cosmic fluid (before the Jeans instability) and in the dark matter halos (after the Jeans instability). This observation may be a hint that the value of the scattering length changes in the course of time and that the scattering length of the bosons in the dark matter halos is not the same as in the cosmic fluid (the density is very different in these two systems, and the scattering length may depend on the density). This corroborates our remark (Chavanis 2011a) that the sign of the scattering length may change, too. Clearly, the determination of the BEC parameters, or the constraints that they must satisfy, is certainly a very important step in validating the BEC dark matter hypothesis.

\section{Appendix C: Equation of state $p=\left(-\rho+k \rho^{2}\right) c^{2}$}

In this Appendix, we consider the equation of state (135) with $\alpha=-1$ and $k \neq 0$. In that case, Eq. (136) can be integrated into

$\rho_{\mathrm{b}}=\frac{1}{3 k \ln \left(a / a_{*}\right)}$,

where $a_{*}$ is a constant. Physical solutions require that $k>0$ and $a \geq a_{*}$. Setting $R=a / a_{*}$, the Friedmann Eq. (82) with $\kappa=\Lambda=0$ can be written

$\dot{R}=\frac{2}{3} K \frac{R}{\sqrt{\ln R}}$,

where $K=(2 \pi G / k)^{1 / 2}$. The solution of Eq. (C.2) is

$R(t)=\mathrm{e}^{(K t)^{2 / 3}}$.

For $t \rightarrow 0, R \simeq 1+(K t)^{2 / 3}$. The curve $R(t)$ presents an inflexion point at $R_{\mathrm{c}}=\sqrt{e},\left(\rho_{\mathrm{b}}\right)_{\mathrm{c}}=2 /(3 k)$ and $K t_{\mathrm{c}}=(1 / 2)^{3 / 2}$. The expansion is decelerating for $t<t_{\mathrm{c}}$ and accelerating for $t>t_{\mathrm{c}}$.

If we introduce the present-day values $\rho_{0}$ and $a_{0}$ of the density and scale factor, the density can be rewritten

$\rho_{\mathrm{b}}=\frac{\rho_{0}}{3 q \ln \left(a / a_{0}\right)+1}$, where $q=k \rho_{0}$. We have $a_{*} / a_{0}=\exp (-1 / 3 q)$ and $K=$ $\left(3 \Omega_{0} / 4 q\right)^{1 / 2} H_{0}$. Using Eq. (C.3) the evolution of the scale factor is given by

$\frac{a(t)}{a_{0}}=\mathrm{e}^{-\frac{1}{3 q}} \mathrm{e}^{\left.\frac{3 \Omega_{0}}{4 q}\right)^{1 / 3}\left(H_{0} t\right)^{2 / 3}}$.

The time at which the universe starts accelerating is $H_{0} t_{\mathrm{c}}=$ $\left(q / 6 \Omega_{0}\right)^{1 / 2}$. The age of the universe, corresponding to the time $t=t_{0}$ at which $a / a_{0}=1$ is $H_{0} t_{0}=2 /(9 q)$ (we have taken $\left.\Omega_{0}=1\right)$. Finally, the present-day value of the deceleration parameter $(157)$ is $Q_{0}=(3 q-2) / 2$.

\section{References}

Arbey, A., Lesgourgues, J., \& Salati, P. 2001, Phys. Rev. D, 64, 123528 Arbey, A., Lesgourgues, J., \& Salati, P. 2003, Phys. Rev. D, 68, 023511 Baldeschi, M. R., Gelmini, G. B., \& Ruffini, R. 1983, Phys. Lett. B, 122, 221 Bernal, A., Matos, T., \& Nuñez, D. 2008, Rev. Mex. Astron. Astrofis., 44, 149

Bialynicki-Birula, I., \& Mycielski, J. 1976, Ann. Phys., 100, 62

Bianchi, M., Grasso, D., \& Ruffini, R. 1990, A\&A, 231, 301

Bilic, N., Lindebaum, R. J., Tupper, G. B., \& Viollier, R. D. 2001, Phys. Lett. B, 515,105

Binney, J., \& Tremaine, S. 1987, Galactic Dynamics (Princeton University Press) Böhmer, C. G., \& Harko, T. 2007, J. Cosmol. Astropart. Phys., 06, 025

Bonnor, W. B. 1957, MNRAS, 117, 104

Breit, J. D., Gupta, S., \& Zaks, A. 1984, Phys. Lett. B, 140, 329

Burkert, A. 1995, ApJ, 447, L25

Callan, C., Dicke, R. H., \& Peebles, P. J. E. 1965, Am. J. Phys., 33, 105

Chandrasekhar, S. 1939, Stellar Structure (University of Chicago Press)

Chavanis, P. H. 2011a, Phys. Rev. D, 84, 043531

Chavanis, P. H. 2011b, Phys. Rev. D, 84, 063518

Chavanis, P. H. 2011c, Phys. Rev. E, 84, 031101

Chavanis, P. H., \& Delfini, L. 2010, Phys. Rev. E, 81, 051103

Chavanis, P. H., \& Delfini, L. 2011, Phys. Rev. D, 84, 043532

Colpi, M., Shapiro, S. L., \& Wasserman, I. 1986, Phys. Rev. Lett., 57, 2485

Dalfovo, F., Giorgini, S., Pitaevskii, L. P., \& Stringari, S. 1999, Rev. Mod. Phys., 71,463

de Bernardis, P., Johnston, J., Mudri, S., et al. 2000, Nature, 404, 995

de Blok, W. J. G., Bosma, A., \& McGaugh, S. 2003, MNRAS, 340, 657

Eddington, A. S. 1930, MNRAS, 90, 668

Einstein, A. 1917 Sitzungsber. Preuss. Akad. Wiss., 1, 142

Einstein, A., \& de Sitter, W. 1932, Proc. Natl. Acad. Sci. (U. S.), 18, 213

Fedichev, P. O., Kagan, Yu., Shlyapnikov, G. V., \& Walraven, J. T. M. 1996, Phys. Rev. Lett., 77, 2913

Friedmann, A. 1922, Z. Physik, 10, 377

Friedmann, A. 1924, Z. Physik, 21, 326

Fukuyama, T., Morikawa, M., \& Tatekawa, T. 2008, J. Cosmol. Astropart. Phys., 06,033

Gilbert, I. H., 1966, ApJ, 144, 233

Goodman, J., 2000, New Astron., 5, 103

Guth, A. H., 1981, Phys. Rev. D, 23, 347

Guzmán, F. S., \& Matos, T. 2000, Class. Quant. Grav., 17, L9

Hanany, S., Ade, P., Balbi, A., et al. 2000, ApJ, 545, L5

Harko, T. 2011a, MNRAS, 413, 3095

Harko, T. 2011b, J. Cosmol. Astropart. Phys., 5, 022

Harrison, E. R. 1965, Ann. Phys. (NY), 35, 437

Harrison, E. R. 1967, Rev. Mod. Phys., 39, 862

Hinshaw, G., Weilaud, J. L., Hill, R. S., et al. 2009, ApJS, 180, 225

Hu, W., Barkana, R., \& Gruzinov, A. 2000, Phys. Rev. Lett., 85, 1158

Jeans, J. H. 1902, Phil. Trans. A, 199, 49

Jeans, J. H. 1929, Astronomy and Cosmogony (Cambridge University Press)

Kaup, D. J. 1968, Phys. Rev., 172, 1331

Khlopov, M.Yu., Malomed, B. A., \& Zeldovich, Ya.B. 1985, MNRAS, 215, 575

Kiessling, M. 2003, Adv. Appl. Math., 31, 132

Klypin, A., Kravtsov, A. V., \& Valenzuela, O. 1999, ApJ, 522, 82

Layzer, D. 1954, Astron. J., 59, 268

Lee, J., Koh, I. 1996, Phys. Rev. D, 53, 2236

Lima, J. A. S., Zanchin, V., \& Brandenberger, R. 1997, MNRAS, 291, L1

Madelung, E. 1927, Zeit. F. Phys., 40, 322

Matos, T., \& Guzmán, F. S. 1999, F. Astron. Nachr., 320, 97

Matos, T., \& Ureña-López, L. A. 2001, PRD, 63, 063506

Mielke, E. W., \& Schunck, F. E. 2000, Nucl. Phys., B564, 185

Milgrom, M. 1983, ApJ, 270, 365

Milne, E. A. 1934, Quarterly J. Math., 5, 64

McCrea, W. H. 1951, Proc. R. Soc. London., 206, 562 
A\&A 537, A127 (2012)

McCrea, W. H. 1955, AJ, 60, 271

McCrea, W. H., \& Milne, E. A. 1934, Quarterly J. Math., 5, 73

Navarro, J. F., Frenk, C. S., \& White, S. D. M. 1996, MNRAS, 462, 563

Oh, S. H., de Blok, W. J. G., Brinks, E., et al. 2011, AJ, 141, 193

Overduin, J. M., \& Wesson, P. S. 2004, Phys. Rep., 402, 267

Pace, F., Waizmann, J. C., \& Bartelmann, M. 2010, MNRAS, 406, 1865

Peebles, P. J. E. 1980, The Large-Scale Structure of the Universe (Princeton University Press)

Peebles, P. J. E. 2000, ApJ, 534, L127

Peebles, P. J. E., \& Ratra, B. 2003, Rev. Mod. Phys., 75559

Perlmutter, S., Aldering, G., Goldhaber, G., et al. 1999, ApJ, 517, 565

Persic, M., Salucci, P., \& Stel, F. 1996, MNRAS, 281, 27

Primack, J. R., Seckel, D., \& Sadoulet, B. 1988, Annu. Rev. Nucl. Part. Sci., 38, 751

Reis, R. R. R. 2003, Phys. Rev. D, 67, 087301; Erratum: Phys. Rev. D, 68, 089901(E)

Riess, A. G., Filippenko, A. V., Challis, P., et al. 1998, Astron. J., 116, 1009

Rubin, V. C., Ford, W. K., \& Thonnard, N. 1980, ApJ, 238, 471
Ruffini, R., \& Bonazzola, S. 1969, Phys. Rev. 187, 1767

Savedoff, M. P., \& Vila, S. 1962, ApJ, 136, 609

Schunck, F. E. 2003 [arXiv: astro-ph/9802258]

Schunck, F. E., \& Mielke, E. W. 2003, Class. Quant. Grav., 20, R301

Sikivie, P., \& Yang, Q. 2009, Phys. Rev. Lett., 103, 111301

Silverman, M. P., \& Mallett, R. L. 2002, Gen. Relativ. Gravit., 34, 633

Sin, S. J. 1994, Phys. Rev. D, 50, 3650

Spiegel, E. A. 1998, in Gravitational Screening, ed. A. Harvey (Springer-Verlag, Heidelberg)

Sulem, C., \& Sulem, P. L. 1999, The Nonlinear Schrödinger Equation (Springer) Takasugi, E., \& Yoshimura, M. 1984, Z. Phys. C, 26, 241

Thirring, W. 1983, Phys. Lett. B, 127, 27

van der Bij, J. J., Gleiser, M. 1987, Phys. Lett. B, 194, 482

Vergassola, M., Dubrulle, B., Frisch, U., \& Noullez, A. 1994, A\&A, 289, 325

Walter, F., Brinks, E., de Blok, W. J. G., et al. 2008, Astron. J., 136, 2563

Weinberg, S. 1972, Gravitation and Cosmology (John Wiley \& Sons)

Widrow, L. M., \& Kaiser, N. 1993, ApJ, 416, L71

Zwicky, F. 1933, Helv. Phys. Acta, 6, 110 